\title{
Siglen= Berzeidnis.
}

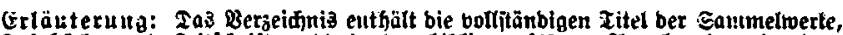

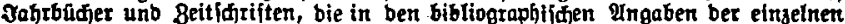

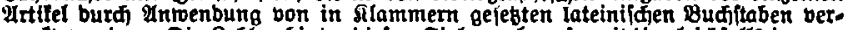
mertt rurben. Die Bahlen hinter biefen Siglen geben, foweit fie gleidffalls in ner.

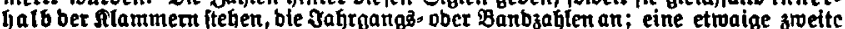
Bafl, ble bon ber eriten burd) einen Quer(trid) / getrennt ift, benennt ble Deftzafil.

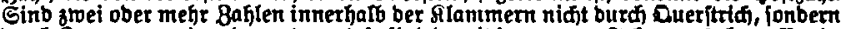

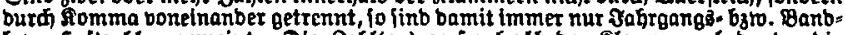
bziv. Seltzahlen gemeint. Dle Bahl(en) au Berhalb ber Jlammern bebeuten bie 3ahreżahl(en).

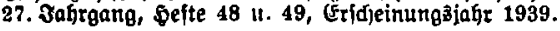

$\Lambda=\mathfrak{U}_{11 \mathrm{th} \text { ropos }}$

AaB $(\mathbf{A c k B O})=$ Ter Yäermautn aui Bobmen

Aach A O Pläjener Rrbjanblungen

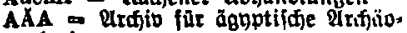
tonie

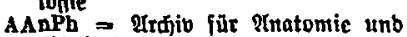
PGyfiologie

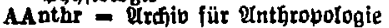

AaS - Urdjiv fưr angetoanbte cozlo. Iogie

AAuH $=$ Irdjiv für Qlugenheiltunbe

AAWW = êlmanad ber Ufabemie ber Bifienifaften in $\mathfrak{B}$ ien

$A B=$ Ingervanbte Botanil

$A B=$ Die \&utobahn

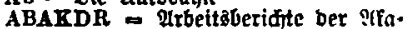
bemte fur Teutifies gredit

$A B b=$ Rrdio für $\mathfrak{B u d j b i t i b e t e t ~}$

A bhadI $=$ Üb̆anblungen aus bem

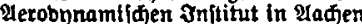

AbhBAw = Mbhartuturaen ber gane:

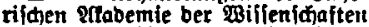

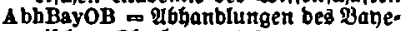
rifdien Sberbergamtes

AbhQWG $=$ Qbhanblumgen ber Geielf. falaft ber खilferidjaften, Gottingen

AbhKin = Vibharblungen für bie R̉urbe bes gorgenlanbes

AbhMSHU $=$ Qbhanblungen aus bem Whatbematibuen Eeminar ber sanfitidien univerfität

AbhMTVD $=$ थbhanblungen bes mu. feums für Iler- unb Bỏtertunbe ou Dreaben

A bhNwGD $=$ 2G6anblungen ber $\mathfrak{N a}$, turtwilien[djaftlidien Befell[d;aft Dresben

AbhowVB - 206janbluntgen bes natur: wollienidaftliden Beretrs Bremen

A bhnwVH $=$ Mbjanblungert bes natutwilfen/djaftlidjer Bereind zu \$am. burg

A bhPrA W (AbhPrA w) = Ubjanblungen bet Breustifier Qftabemic ber शiti. fenifjaften
AbhprgLA - Qbfjanblungen ber BreuBilifien Geologiffien lanbesantitalt

AbhPTRA = 296hanblungen bex 356 .

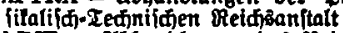

AbhRW = Ubsanblunger bes Reld) amt8 für 2 etterbienit

AbhaA w $=$ 2rbhanblungen ber Gäd)iifien artabemte ber miflertidafter

AbbSNG = Qubsanblungen ber Eendert bergifíen Paturjoridjenben Geiell: ifalt, Franthurt/m.

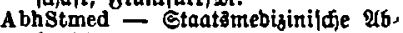
hanblunger

$\triangle b$ bVWG $=$ शбhanbluttgen utb Borträge ber Bremer $\mathfrak{B i}$ ifen|djaftlidjen Gieieltiolaft

AbhWestlalPrM $=$ 2nbfanblungen altz

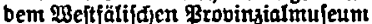
Mtünftet

ABlen - VIrdio für Sienertumbe

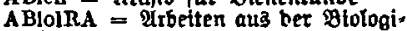
\{djen Oteidjantitalt

A Bioth (ABth) = Acta Blotheoretica

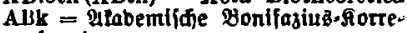
ponbenz

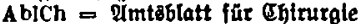

AbiRminW = Vimtöblatt bes 亿eld)o. minifteriume für galifenifjaft ufm.

ABRA = Qurbelten aus ber Biologiffien Beidjöanitalt

ABStM $=\mathfrak{u}$ mtlidje Berlajte ber Staat. Iidien Diujeen

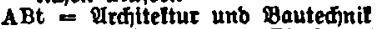

ABth (ABloth) - Acts Blotheoretica

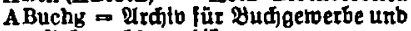
Gebraudjegraphit

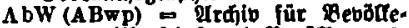
zungsిmilienidjaft unb bevobltenungs. politit

AC = Untile unb Ehriftentum

AckBo $(\mathrm{AaB})$ a Der Qdermann atto $B 03$ men

$\Delta C l$ (Antclass) $=\Delta$ Antiquité Classique, Bralftel

Act I $=\Delta$ cts Mathematlco

AD = IItrjib fïr Dermatologie 


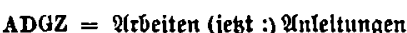
ber Deutífien Gelellidjaft für Bith tungötunbe

$\Lambda \mathrm{dH}=$ शTS ber Seimat

$A d N=$ Yus ber ylatur

ADSw $=$ Á Eectuarte

ADSy $=$ Qtน̆iv für T crmatologie un ธิyphtlia

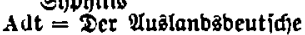

AdVI = शtuslanbbeutide. Polfsfor \{कுung

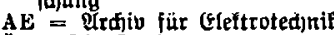

$\mathbf{A}=$ Die arztin

AblBay = Arsteblatt fïr Baijern

Aeg = Aegyptus

$\mathbf{A E G M}=\mathbf{A H G}=$ olitteilungen

AFHW $=$ शrdjib für bas cifentiutten iveien

A Fis = Qrdfiv für Gijenbahnueien

AELKZ $\bar{B}_{\mathrm{B}}=$ थIlgeineine Guangelijd): Sutgerilidie firdienzeitung

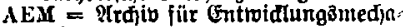
itill

AEmO $=$ Qrdjiv für Githoidfung: unechanif ber Srganisutuen

$\mathrm{AePPh}=$ Urdit für experimentell \$atfologie unb \$harmalologie

IrztlPr = Argtlidjer Brattiter

IrztlR = Irjtlidie Runbidialt

ISZ - Rrgtlid)e Eadiocritünoiben3eiturty

AevKR $=$ :(rdjiv fïr evangelifios Sirchenredit

AEVLKZ $=$ allgenteitc Foangilitit: qutberijdge sirdjenzeitung

$1 e Z=\mathcal{Q}$ r $\dot{\omega}$ iv für experinentelle $3 \cdot 11$ forfdiung

$A F=$ Die Uniकlubfrage

AFbFI. $=$ Prdjiv für bie jortid)ritte betriebänirt|djaftlid)er for\{djund unb Qehre

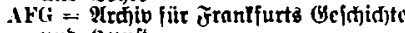
unb sunit

AFUL $=-$ PIrhiv für Franffurter (Sefifidte unb Lanbegrunbe

AFH $=$ Archlvuin Franciscanun Iistoricum

AFJz = Nugenteite forjt. unb Jagb. Beitung

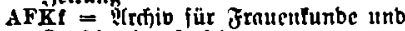
Sonftitutionşorfidjung

Arr = Africa

Afr $=$ Ufrila.stadididen

AfrR $=$ afrila. Runbí)au

AFZg = भIgemeine Fiff)erei-3eitung

$\mathbf{A g}=$ Der Ârbeitgeber

AOAO = Arthis für (Geídichte unb Ultertumatunbe vol oberfranfen

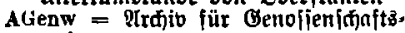
toefent

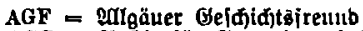

$A G G=$ बrăfib für (Bewerbepathologit unb Gerverbehngtenc
AGHA = Mrdity für Gefdjifle toe

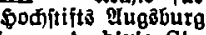

AGlu = Archivio Gluridlco

AQkd $=$ Qtrđ̆iv für Geflügelfuntoc

AGLuBO $=$ शbhanblungen ber Geolo giffjen Lanbezunteriud)ung ant $\mathrm{Day}$ rificst Eberbergamt

AGM = Qrafib flir (Sejhidite ber Dle bizin unb Raturtwilfenifiafter (Eub Goffo Q(rditu)

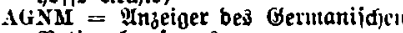
Rationalmuleums

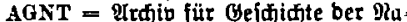
turtoilfenidiaften unb ber Tedinil

AGpGh = Qrtajib für Getverbepatholo gie urb Gemerbchngienc

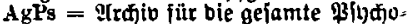
Iogite

AGT $=$ Qrđjiv für Getriebetedittit

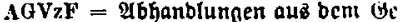
biete ber Bogeljugforfing

AGWG $=$ abfanblungen ber Gejell

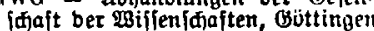
AGyn = Irffiv für (Snnälologie

$\mathbf{A H b}=$ भrafib für Storobiologie u॥ \$lanttontunbe

AhOAk = Orofiv für hefiifole (Bejđjiffte unb शrtertumtôtunbe

AHIR = Qbhanblumgen bes̄ jerber Jnftituts $\mathfrak{R i g a}$

A HMS (AMSB) $=$ Ubbartulugen aus bem Mrathematificen Geminar ber ธantburaif́en univerfitäl

$\triangle H N O=$ Urajiv für ఏalä, Rafer, Efrentincintube

AhVNrh = Innalen bes biftorijuest Sereins für ben liebertheir

$\mathrm{AHy}=$ Q(rd)io für sugiente unb saftc. riologie

$A I=$ Ars Islamica

AICh $=$ A rchivlo Italiano di Chirurgia

A $\mathrm{Kol}=$ Irchiv für innere হ̃olonifation

AIM $=$ Archives of International Medicine

AIVKrb = Acti Jntentationale $23 \mathrm{cr}$ cintigutg für Stebałforidung

AJM $=-$ Americall Journal of Mathe. matics

Ak $=$ Die Ifobeutie

Akathvbl = OIthatholifdes ßultsblut

$A k B 1=$ Qtabemi (千)e Blätter

AKG $\left(A_{\mathbf{K}}\right)=$ QIrfib fïr stultur. gefoifite

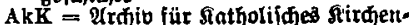
redit.

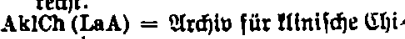
rurgie

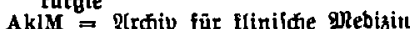

AKrF $=$ Q(rd)iv für Sreislaufforfdutt

AkThBl $=$ Qthabemidaje Theologijd) Blätter

AkuZ $\Rightarrow$ ertujtiffije Beitidriłt

$A L=$ शrafib für Laryngologie

Al $=$ शluminium

AlpMrh = शttpentänbijific Mronatōljefto 
ALstF $=$ Urdiviv für Sancritättenfor idung

AltH $=$ घht oiloç̄heil

Altschl = Mltíflefien", Mitteiluntgen

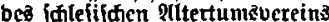

$A L V t=$ Qudfio für \&anbes- unb 3 ulf: forid)ung

$A L w=\mathscr{Q}$ rdiv für Ranbwirtidait

$\mathrm{Im}=$ Acta mathematica

AmA $=$ P(rdjio fïr mifroffopild)e ?itutomic

A Mat = Acta di Matematica

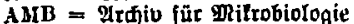

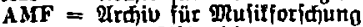

IMGIStU $=$ U6́janblungett aus bell

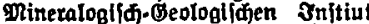
ber Єt. Tizza.llniberfaüt in Debrec. It

IuNT = Qutdio für Mathemait, Raturtviffenfjaft urb זechitil.

Amp = Annales medico-prychologlques

AMSI (AHMS) $\rightarrow$ Mbhanblungen aus bem Prathematifjen Geminar ber Somburgíácen liniverfität

Amus = Acta musicologica

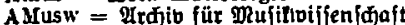

AMLEZ = 2rIgenteine Mrufit. Beiturg

ANw $=$ Inzeiger für Mafijinentoejet

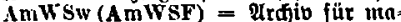
thematipdie Mzintidjafta- unb Eosial foridung

$A N=$ Q(ftronomilife gad)ridite!

AnA $=$ Inatonififfer IInzeiger

AnBosStIemb = QInnalen von ber llos scha Sterrenwacht Lembang

$\Delta N_{g}=\mathscr{A}$ rdiv für Naturgeidinite

Ang $=$ Anglia

AngBot = QIngetuanbte Botanit

AngCh $=$ Slitgetoanbte Themie

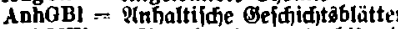

AnhMW = IInnaler bes naturhiftori

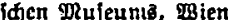

AnnCh (LA) $=$ Prnalen ber Shemic

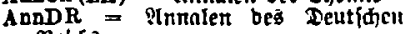
Reldiaz

Anulydr = 2nnalen ber snbrographic unb maritimen Reteorologie

$\Lambda$ nn myc $=$ Anoales mycologic

AnuPa = Annales de Parasitoloyio

AnnPh (WledAnn) = Intralen ber $\mathfrak{B h}$ gfit

AnnPhil = Pinnaler ber Bhilolophie

Ant $=$ Die Antile

Antclass $(\mathrm{ACl})=$ Antiquite classiqute (Brülfiel)

AnthrA $=$ Untfropologijdyer Anzeiger Aöfll = Profiv für offentliges Otent $A O F \Rightarrow$ Rrdjio für Drtentforf氏int

$A O G B=$ enteiger ber Denithologilnet Geferlifialt in ganem

AOh $=$ Q(rd)iv für Ehrenflifturoc

Aol $=$ Acta otolaryngologica

AONK $\Rightarrow$ शrdiv für Ehrett, 9iajel und Rehltop fheiltunbe

AOr = Analecta Orleatalia

IoUCh $=$ Urdfiv für orthopäbiidı ult Hnfall-Ehiturgie
APANL = Atti Pontifichl dell'Accidemla Nazlonale del Llncel

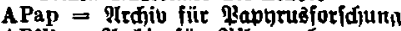

APfB $=$ Qrdfiv für Qjilanzenbau

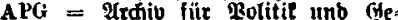
fdidite

$\mathrm{APh}=$ Archlvium Phllologlcum

APharm = Urofiv ber Bharmazte ulb Beriकte ber Deutidien Bharmajell. tifj]en (Helelliqjaft

APhilscand $=$ Acta Philologica Scan dinavica

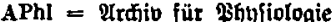

APhs $=$ Urdilo für Bhilofophte unb Gojiologie

APhysP $=$ Acta Physics Polonicn

Apr $=$ Mttpreufen

AprB $=$ Olttpreu Bi

AprF = 2Mtureubifale forldungen

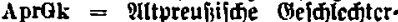

funbe

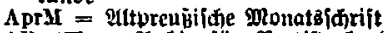

AProtK $=$ P(rđ)iv für Brotiftentuntoc

AYs = Acta Psychologica

$A P s N=$ Irøị für ßingitatrie uno Rerventrantheitelt

A pZg $=$ QLpotheler* Beitumg

A RassB $=$ Urffiv für Mrafienbiologie

ArbIeTh = Nrbeltei auş bem 3nftitut für experimentelle Therapie.

ArbK = Der Ürbeitătamerab. Betriebs: zeitfidrift ber (Commer3- unb \$ribat. Pant

ArbPh = Qrabeitøphyffiologie

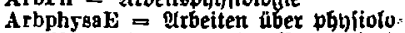
gifine unb angetwanbte Centomologic

ArbRs $=$ Q1rbeitorect unb Ghlintun

Arbsch $=$ Die Arbett3|quule

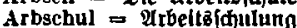

ArbuG $=$ Wrbeit unb Gejumbheit

Archacol = Archaeologica (diopci). hagent)

ArchAnz $=$ Irdjäologiidjer Inseigcr ArchEph $=$ Archalologlke Fphemeris (2then)

Archl $=$ Archltecturs

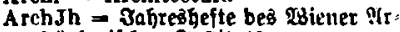
djdologi [óen 3ititituts

ArchR $=$ Archlwum Ronauletu (Berf- florens)

Archst $=$ Undivitubient

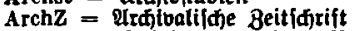

ARGA $=$ Urbeltet aus bett Reings. nefunbhettäamt

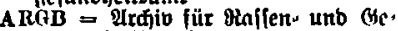
(eII)dajtsbiologie

Arkiv $=$ Arkiv för nordisk Fllologi

ArmB $=$ Qrmietter Beton

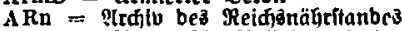

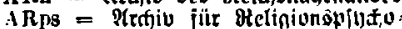
logic

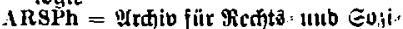
alphilofophie

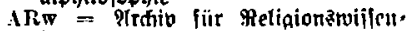
ithaft 


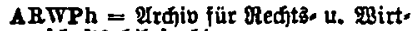
idjaft żphilolophie

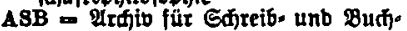
toefen

ASchB] = 2ntt\{filefiline Blätter

A Schl (Altschl) - थltiolefien

ASchMz $\Rightarrow$ Allgemeine Edpweizerifoje DTilitärz eiturn

A SchTHy $=$ Urđfiv für હđiffż unb Iropenhygiene

ASD = Albatarios Studia et documents

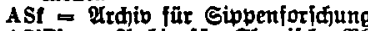

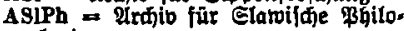
Iogie

Asm $=$ Asla malor

$A S 02 W=$ Rirchiv fitr Eojialroilfent idaft unt Єozialpolitil

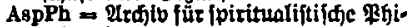
lojopfie

Aspr $=$ AIte Gpradien

ASpStaPh $=$ Ardíto für Spraç- unb Stimmfeilfunbe unb angeloanbte Bhonetil

Asta $=$ argemeines etatiftiffes श्रrofito

AStHG $=$ ঐrdjiv für Stubenten- unb

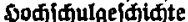

AstaSpr (Berra) $=2$ trujiv für bas Etubtum ber neueren Epradien unb Qiteraturen

AStR (GoltdA) = 2trdito fût Gtraj, refit unb Strafprozes

ASwSp $a$ Utdity für Sugialwifienidjaft uno Soglalpotitil

$A T=$ Qfiphalt unb Ieer

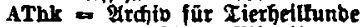

Athifltt (MD ATA) = Pritteilungen bes

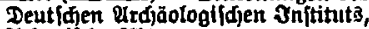
Utheni đue थlbt.

Atl = Qutlants

ATMI = Urdib für tedinildes Meifen

ATz o थrofib für Tteremährung unb Tiersudit

AUF - İrdito für urfunbenforidiung

$A u Q=$ Urbett unb Gemeinfijaft, Blätter für Qurbeitsbeldiaffung

AuMet $=$ Qutogene Metalibearbeitung

AOR = Qurdito für Urgeber., Film. unb Theaterredt

Ausl $\Rightarrow$ Die Plustefe

AuslFt $=$ Qlusiele ber Funitedinif

AusiT $=$ Dle Quuâlanbsitedjnil

AutB = Die Qlutobabn

AutSch 2 Der Uutogen|djtoeífer

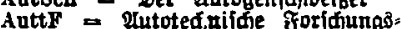
arbeiten

AuttZ $\square$ 2utomobiltedififi)e 8eit. fかitift

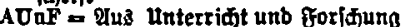

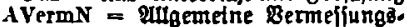
Tadjtiditen.

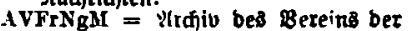
greunbe bor Maturgeldidite in Medlenburs

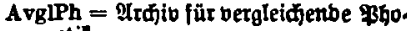
netil

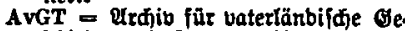
(d)idite unb Topographie

AVIrK $=\mathscr{A}$ rafivi für Biruşhurbe

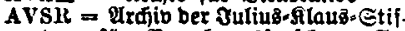
tung für Bererbungeforifiung, Sv zialantbropologie unb ofaffenhybient

AWandw $\Rightarrow$ Yrditb für gBanberung weien

AWD $=$ Qrafil für gBärmetoirtianait unb Dampltelíelbetrieb

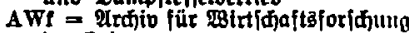
im orient

$A W n p G=$ Iuf bem mege zum natio nalpolitifífen Gnmnaltum

AwprT (AwpTh) = Qrdiv für wiffelt ¡jaftlidje unb praltijoje zierheil. tunbe

AwpTh (AwprT) $=$ Qrdit für tviffen (đ)aftlide unb prattifoje Tierbeil. tunbe

$A W R=$ Urđjiø für Bettbelverbòred)t

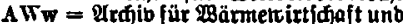
Dampfteffelivejen

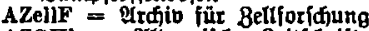

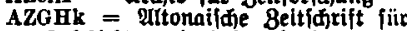
Gefdidfte unb seimattunbe

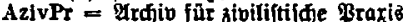

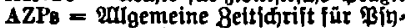

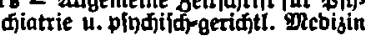

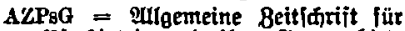
Pindiatrie unb ince Grenzgebiete

AZPsth = ellgemeine geitifirift fur Bibdiotherapie utu pindilide sy. giene

$\mathbf{B}=$ Die Püđjetei

BA $=$ D. Bant Qurfib

BAA = Dertate bes Mitien-2rbelt treiles

BadFb = Babifide Funbberifite

BadSch = Dle babtifie Sdite

BEYr $\Rightarrow$ Beiträge zur ärstliđen \$rar is

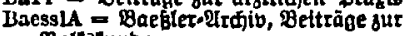
Bollatumbe

$\mathrm{BaGb}$ - Bauamt unb Gemelnbebau

$\mathrm{BaGPh}=$ Betträge zut angetwanbten Oeophyjia

BAISt $=$ Bollettino della Accademia Italians di 8tenografia

Baln a Der Balreologe

Balt3ith = Baltiluge Dionat

BAMS - Bulletin of tho American Metcorological Soclety

Bank = Die Bant

BAPhO = Belträge zur Unato mate, Phy. fiologie, Batbologle unb Theraple ber Chren, ber Maje unb bes Sallea (Bajlom.S̄tiaefer \&eiträge)

BA $\mathbf{K}=$ Bibliotecs dell'Archivin $\|_{0}$. manlcum

BauG = Das \$augetwerbe

Bauing = Der Rautngentells 
Baum = Der Moumeifter. Ponatâ. hefte für Baulultur unb Baupraria

MauSch $=$ Bautenichus

BauT = Die Bauteकnil

Bauw = Die gaumarte

BayBw = Bayeriĺtę gitbungätweicn

BayGVZ = Bayeri ige Gemeinbe- unb Bernaltungs. Beitung

BayHSch $=$ Bayerifier Seimatíubs

BayHVk = Bayerijđfc פefte für Boltz: tunbe

Bayld (Bld) $=$ Das BayerIanb

BayLZ = Banerif́fe Lehrerzeitung

BayVHI $=$ Babetifde Bervaltungä blätter

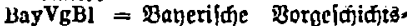
blätter

BBBotG $=$ Beriate ber Bayerifojen Botanilinen Gelellifjaft

BBlf KG $=$ Samberger Şlätter jür fräntiloje sunft unb (seididite

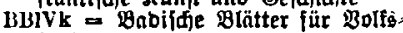
tunbe

BlNw = Brejner \&eiträge zur Yatur twillerifiaft

BBotZ (BhBZ) = Seifefte zum 2in tantidien Bentralblatt

BBPfl = Beiträge zut Biologie bir Dffartzen

HBRPh = Werliner \&eiträge jur ro manildien ybilologic

BbwA = Berifte über betriebsibilicn. (d)aftlide girbeiten

BChIns $=$ Heiträge jur Shemic bes Jniulinz

BchPhysP = Beiträpe zur dieunifdien Bhyliologic unb \$athologie

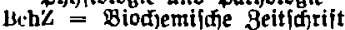

LDBU = Serigite ber Dcutiofien $2 \mathrm{O}$. tanifdicn Gefellidjait

ISDChG = Berinte ber Deutidjen Elje. milden Geielilifaft

BDKG = Beridte ber Teut [djen Jicras miifien Beiclifigaft

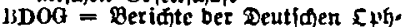
thalmologijáfen Gejellifiaft

UDR - Bullctino di diritto romano

HDwKMI - Betidte ber beutfuen roiffenifiaftliden sommiffion für meeresforidiulg

Buba = Det bergbau. Bergtedjnijuje

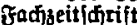

Belv - Belvedero

BerRGK - Beriate ber $\Re$ domi ifj-(Ber manifien stommiffion

BetEis = Beton unb Cileu

HetrW = Die getriebsెivifienifiaft

BetStr $=$ Die Betonftrafse

Bew = Bemegung

BFBV = Beiträge zur fortvflanzun biologie ber $\mathfrak{B}_{0}$ öel

BFNG $=$ geriffte ber frteiburger gia turforfienben (Bejelliffajt

Bgb $=$ Det Berobau
$\mathrm{BOBr}$ (BGStBr) = Beiträge zur Gefiftite ber etabt Brealau

BOBuGg = Beiträge zur obttinger ubibliothetz. unb Gelehrtengefininte

BGdS (BGdSpL) = Beiträge zur Ge [didite ber beutidien Spradje unb Riteratur

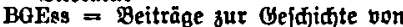
Gtabt unb Gtift E[jen

$\mathrm{BgG}=$ Beriate liber bie gefamte-(5t)nätologie unb Geburtăhitfe

BGH $=$ Daubener Beifiditishefte

$\mathrm{BgPhl}=$ Betidfte über bie gefamte Phyjiologie

BGTh = Peiträge zur Oeologie von Thüringer

BGTI = Weiträge sur Beididite ber Tedinil unb 3nbuftrie

BGVM $=$ Beiträge sur Geزfjidte ber Beterintär-glebizin

BHAKB = Beiträge zur Seimattunbe

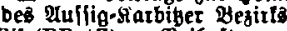

BhBZ (BBotZ) = Beifefte zum Bota. nifidien Bentralblatt

BHHFK = Beiträge zur Gauto, \$narunb Felftunbe

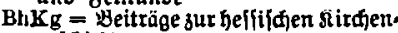
geiditute

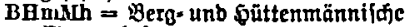
Dionatsheitc

BHsch = yeamtenfodidule

BHVB = Weridte bes siltori[den Ber. cing Bamberg

$\mathrm{Bi}=$ Dag $\mathrm{Hilb}$

$\mathrm{Bib}=$ Biblica

BiblZ = yiblifhe Bcit/dirift

BICHS = Bulletin of the Internatlonal Cominitteo of Historical Sclences BIDR $=$ Bullettino del Istituto dI Diritto Romano

BIGK = Beriate bes Internationalen (ทiейerei-si ongrelles

BInk K $=$ Beiträge sur \$nthunabelhunbe

Bloch $=$ Die Biodjemie

BioklBb] = Hiollimatilife Beiblätter

Blul = Lex 2iologe

BlulB = Eiolugifine Beridit

Blulgen $=$ Blologla Generalis

BlulRe $=$ Blologlcal Reviews

Blolz = Biologildes Bentralblatt

Bit $=$ bitumen

$\mathrm{BIZ}=$ Biblijhe Beitínrift

Bk (Braunk) $=$ Brautlohle

BkF = Beitröge zur tontmunalen $\mathfrak{F i}$. nanzwirtidjaft

BKIG $=$ guIfetin beş somitees für

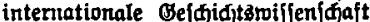

BKI - Betlinet Allinit

BkICh $(\mathrm{BrB})=$ Brung' niniffen Crinuraie

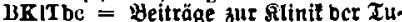
berfuloie

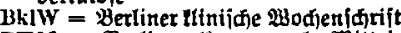

BK $\mathbf{M} \Rightarrow$ Berliner Sinmunale 9 itte lutraen 
BkPfLE = Die Bobentunbe unb \$BIIan. senemährun

BL $(B L w)=$ Reriffte ïber Rnmbwirt. 俰alt

BIBB = Blätter bes Sayreutfjer Punbez

MIBLVI = Blätter bes Banerifjelt Lanbeżbereins fïr Tramilientunbe

Bld (Bayld) $=$ Ias Yayerlanb

BldLA $=$ Blätter für beutldhe Lanbea. geifidite

BIDVg $=$ \&lätter für beutiðje $2 \mathrm{vr}$ geidichte

J3]dPh $=$ \&lätter für beutjuc \$gito. jophic

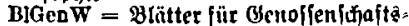
ivejer

BlGk $=$ \&lätter für Gejütuntątunbe

BlHk = glätter für Seimattunbe

BlMfr = Blätter für Mrünsizeunbe

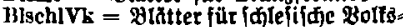
tunbe

BISchprEw = glätter für bie క̄đful vraris unb Grztehungsిmilíenidialt

JiVkMIv = Blätter zur Lanbe s uno Bollötumbe be giththiertels

J!VMI $=$ Blätter für Berídierungs Mathematil und verivanbte Gebiete

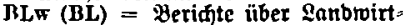
idajt

BIwKG $=$ Blätter für nürttemberglidic Rirdiengeifithtc

Bm - Der Baumart

BMDBl = Betliner IRünzblätter

BMh = Bexlfner gmonatōhefte

BmLbw = gitbincfiung unb Quftuilo. ivefen

BMSNT $=$ Beriate bet mu[ilidule Reu-Titidietn

H.MtaW $=$ Đerliner unb $\mathfrak{P}$ ünthener

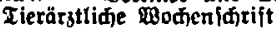

BMus $=$ Berliner Ruleent

BMvorg AK (Olfa) = Effa, Bcriate

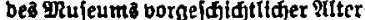
tümer in Riel

linkFSwD = Beiträge zur naturlunb.

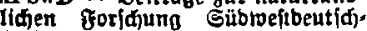
Ianba

$\mathrm{B}_{0}=$ Das Bollivert

BodBu = Bör [enblatt für ben beut伭en Bud baribel

BOG - Drondoftopie, Delopfagoltopie, Gajtroilopte

BOhaNH = Beridjte ber Cberbelififien Géellfidaft lür Natur- unb Đeil. lunbe

BonnJ = Sonter ßabrbühjer bes 2er, eing von 2lltertumgreunben int Mhelitianb

BonnZThS $=$ Bontce Beitifijtijt fïr Theologie unb Seelforge

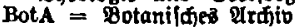

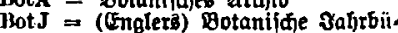
fier fur Gyitematit, Bflansengs. ifhidte unb gflankenneographie
BotZ = Botuniidjes Bentralulatt

BpAaP (ZiegBl) = Reittäge zur vatho logifien elnatomic unb jur allgc incinen Bathologic

BPfl (ZPflD) = Pobculunbe unt Pflanzenernährums

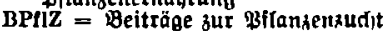

BPhDI = Beiträ̉e zur Bhitofophie oc Deutígen Toealiantua

BPhfrA $=$ Beiträge zır $\$$ hy fil ber freic Q1tmołphäre

BPhmed SE (SBphmSE) $\Rightarrow$ Werioft (Eibungaberidite) ber whnfitaliid) mebizinifijen Eozietät zu ErIangen

BR $\Rightarrow$ Beittäge zur Pedtbernetic rutn

BrandJb = Sraibenburbijific InYir bǘfier

$\operatorname{Braunk}(B k)=$ Sraunfoble

BrB (BklCh) $=$ Brură Beiträge zu Ilinifjen Chinurgie

BrCh $\Rightarrow$ Brennitoff-(Shentic

BRF = Beridite zur Runenfurfäun

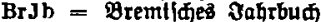

BrPrF (FBrPTG) $=$ Branbenturgi/d) Breubi iøje Foriđungen

BrWP (BWp) $=$ Braune $\mathfrak{W}$ irtidjaltapoft

BSB $=$ Siłunaz̄berióte ber Brcu is. Ultab. ber Biflenidiaften, Berlin

BSch $=$ Die babifhe Ediule

BSEHN $\rightleftharpoons$ Boletin de la Socledad Es. pañola de Historla Natura]

Rstd = Bauemitanb

IBStBA $=$ Beriate ber Stubienaciell.

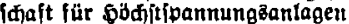

BStr = Sic Betonitrafe

BstWw $=$ Brennftoff = und $\mathfrak{M a ̈ r m e t o i r t : ~}$ idjait

BStZ $=$ Betoriteinzeitutro

BtвW $=$ Berliner tleräratlidje : 3 odjen . (d)rift

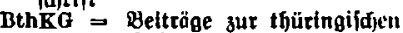
תirdjengef fifite

BTMI = Dertate über bie ₹ätinleit bes Meteorologildien $3 n$ [titut

BtW $\Rightarrow$ Die Betriebsmintidaft

BuE $=$ gilbung unb Eraiefung

BuBpll = güderei unb gitbungâtflege

Büch $=$ Die Púfietel

Bukk $=$ Bühertunbe

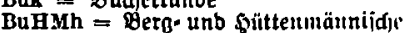
Ronatşbefte

BullsEV $=$ Bulletin be $\epsilon$ fitveiser Glettrotedinifinen Bereins unb beit

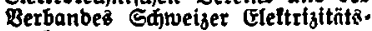
toerte

Burgw $=$ Der Burglvart

BursJ = Burtan Ұahreaberid)t übcr

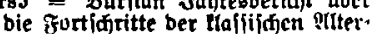
tuutstwifienifiaft

BuS $=$ Bud unb Earrift

BuZ = Die Biene unb ifte Budst

BVschorn = Beriate beß Berein inflelifóner Drnithologen

$B \Pi=$ santruilfenifiaft 
J\}wBl = Bettiebōlvirtidgałtlige slätter lsWp (BrWP) Braune $\mathfrak{k i r t}\{$ djajt3pojt

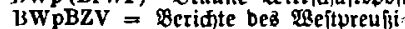

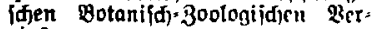
eins

Bwt $=$ Bautvelt

$\mathrm{Byz}=$ Bysantion

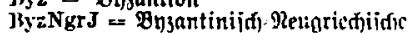
Jahrbüđjer

Byr: $\angle \Rightarrow$ Bysantiniiafe Beitintift

BZAW = Oeibefte jur Beitjofrit jür QItteftamentlidse sisilieniafiaft

\section{$\mathbf{c}=$ Caucasica}

Ca $=$ Caritas

iar = Carinthis

lath $=$ Catholica

(CelCh $=$ (sellutojed)enic

(hA = chemildie \&uvaratur

ChE = Ghemie ber Grbe

ChEJ = Chinese Fconoinir .Juntua

ChF $=$ Tie Chemilde F̂nbrif

('hir $=$ Der Efinurn

ChL $=$ Qlua bein Ohcliner Saiti

Chr(t $=$ Dic (Efriftennenteinitidait

(hrK $=$ Creititlidje sutnit

ClruW $=$ (Shriftentum unb wisigniffuit

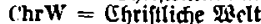

('hU = Efemildje llmifful aui bem (Scbiete ber Zctte, Ele, Lilad)ic umb varze

chy $=$ Ehemiigies sentralblatt

ChZg $=$ Ehemiler-3eituntu, söthen

Ciba $=$ ('jba*Beitfartift

(ic $=$ Clccrone

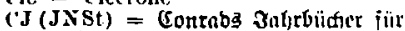
शationatölonomie unb Etutiistif

Coll $\Rightarrow$ Colleaium

Comaue $=$ Commentarli mathemat ini helveticl

(ompjy = Compositio Mathematlea

ComVlad = commentationes Vindobn nenges

Cor $=$ Corons

(r $=$ Crebitreiorm

Crf $=$ Comptes rendus fraucuis

ClRP = Comptes Rendus Paris

CWiss = Ehriftentum unt Esiifenidjuit

Cyt = Cytologia

DA = Icutije थrbcit

DAbi = Deutifies Frateb!ntt

$I D Z g=$ Deutide ofrzte:3eituny

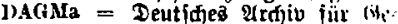
iffid)te bes mittelalters

1) AgrP $=$ Deutidie Q1 ararvolitif

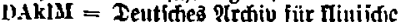
Mebizin

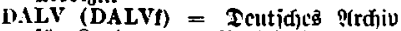
für Lanbes: unb Boltsforfdum

DAPZ $=$ Deut lofe Ivothelerzcitung

DAR = Icutific פenbemtioge Rumb. 低化

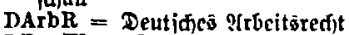

NRanW = Deutific Bautuelet

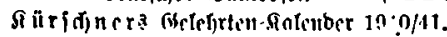

$\mathrm{DBE}=$ Die beutide Herufšcrsichung DBh $=$ Deut[đe Saukütte

DBIH $=$ Teutíd) $\times$ Brafilianifier $\Im_{n}$, buftrie- unb sanbelgführer

DBiw $=$ Deutines Bilbungs̄weict

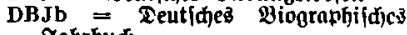
3ahrbudi

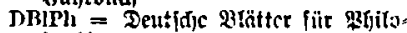
fophic

DBIPo $=$ Deutide grättcr in Solen

DBW $=$ Deutide Bantwittialait

DBwZ $g=$ Deutide Bergluerts. Beitum

DBZg = Deut fqe Bau= Beitung (Berlin)

DChrist = Deutifiez Chrijtentum

DE $=$ Der Deutide Grjieher

Dech $=$ Decheniana

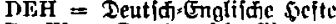

DeuW $=$ TaB beutiffic Mort

Deva $=$ Devilenardiv

DlivFrz $=$ Deutifie (Evangelijde (5r. ziefung

DEZ $2=$ I'utidje Entontologildje 3eit ificiit

$\mathbf{D F}=$ Ientide forfoung

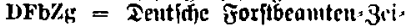
tititg

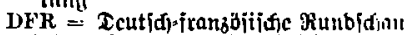

DFrö $=$ Dentfáje Frommigteit

DFW $=$ Ter Deutifíe For ftuvirt

DF $w=$ Tie Deutifie Fertiguarc

DFZ $Z_{5}=$ Teut idje Foritzeitung

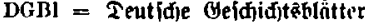

DGl $=$ Deutider Glaube

J)gn = Tie Dorfgemeinfífait. Mronat. iffriit fitt Lanblunbe unb Iänblidic Erjicljung

DFirL = Deutide GrensIanbe

DGWR = Deutidjes Gemeill wis

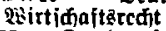

Dli $=$ Der beutidie servio

DHIHI = $\mathfrak{T a}$ Deutidje fanbiverfitifatt

DHdw = Deutífic s Sanbivert

DHöSch = Iic Teutide süferc Efjule

DHLf = Dic Dentide Spilnilange

DHT = Dansk Historlska Tídeskrift

Dily = Deutidie sefte für orlt's forid)ulta

DIVKf $=$ Deutidie Gefte fiir Bolls . "tto গิutturbobenforidjuna

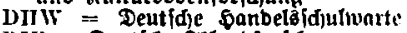

DIF $=$ Deutífe Zalanbforifung

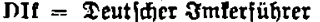

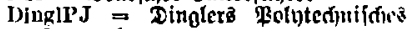
ชournal

DiplI, = Der Diplomlanbwitt

DJa $=$ Deutíaje 3agb

DJb = Denbrologifádez̧ Zafurturi

DJh $=$ Deutidie Jugenohilfe

DJu $=$ Deutífe $\mathfrak{\Im} u$ It $^{\prime}$

DJZ = Deutidye 3urijten - Reitunty

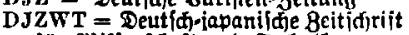

für gisijien|đjaft unb ₹eđ)nif

$\mathrm{DK}=$ Der beutide faufmann

DKch = Deut/he Sieferthinurnic 
DKDP = Deutide ริunjt. unb Deni. malspflege

DKF $=$ Deutide Siraftfahrioriøung

DKkZg = Deutifige Grantentafien

DKLV ${ }^{\text {geitung }}=$ Deutide sultur im Leben ber Bölfer

DKolD $=$ Deutifier Sotonialbienit

DKolZtg = Deut ifie Folonial-8eitung

DKS = Deutifie furzifitif, QRortats. idrift bcr beutjifen Etenografen. qٔiaft

DKultw $=$ Deutige fittur-2Bodje

DKw = Deutide STultumondt, slätter

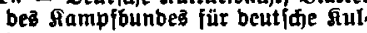
tur

DLGZ = Ieutidje Lanbivirtíjajtlidje Beflügelzeitung

DLR $=$ Deut [ne Sebensinittel-Muno |千̆au

DLwGenBl = Deutifies Ianowirtifjalt linges Genvifenidjaftablatt Brag

DLw:GZ = Deutide Lanbroirtianaftidie Geflügelseitung

DLwPr $=$ Deutide Ranbwirtiajaitlidie Breffe

DLwT $=$ Deutifie Lanbrirtidaftlidje Tierzudit

DLZ = Deutide Riteratur. Beitung

DII = Deutlife Drathematil

DMa $=$ Der beutidie militärarat

DMaZ = Deutífe Metallarbeiter 8cituus

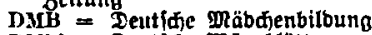

DMbl $=$ Deutí่e Mrūnzblätter

DMhP $=$ Deutifie Monationefte in Polen

DMinwZ $=$ Ientifie minteralwalfer zeitung

DMK $\square$ Deutide פhithfultur

DMoZ $=$ Deut/je פonatgidrift für Batnheilfunbe

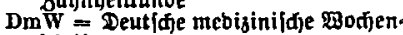
fínift

DNJb $=$ Deutif. Rorbifines 3ahrbudi

DNmR $=$ Deut|d)e RahrungsmittelRunbidjau

DNotZ $=$ Deut [d]c RotarB.8citíntit

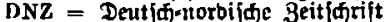

$100=$ Icr Icutidie im Ditrn

DÖk = Der beutifice Olonomit

DörV = Deutiaje iffentlidj-redftlidje Ber[idierung

DPIB] - Dentíajes \$farrerblatt

Dpfl a Dentmalpilege

DpflH $=$ Die Dentmalpflege in ber Brobins Sarnover

DpfiHsch = Denfmalpflege unb פeimat\{jus

DPz = Der Deutidic Felstierzliditcr

DR = Deut|ces Oreht

DRev = Deutfije Brebue

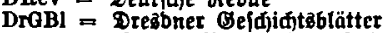

DRIHI $=$ Deutíf.Plumäntífjer Snbu. ftrie= unb ganbelą̧üfirct
DRPI = Deutide Mectiōyflege

DRsch = Deut jie Oumbifiau

DRw = Deutidie Reditäwillenifjaft

DKZg = Deuthie Dithter. Beitung

DSch $=$ Die Deutidie Equale

DSchA = Die Deutidje Edjule im S1n: tanto

DSchAWW = Denlifififten ber $2 f_{1}$ bemie ber miffenidjaften mier

DSchbl = Deutiaje Sđjađiblätter

DSchlZ = Deutine Sdladthof-3ei turg

DSchzP $=$ Deutidje Erijułgeitung il Bolen

DSpoZg $=$ Deutide Gportartifil. Beitung

DSpwZ = Ieutific Evielvarenteitunn

DSSch $:=$ Die beutifie Gonberifjulc:

DStenZg = Teutidic Etenographeu Beitung

DStR = Deut ldies Strafredit

DStZ $=$ Deutidie Etrafred)tz-BeituIn

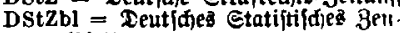
tralblatt

DT = Teutiđie Teøinil

DTäBl = Deut fófeả Tierärzteblatt

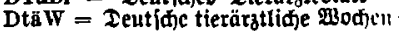
idirift

DTB $=$ Deutíner Tabalfaul

DTbcBl = Deutífez Iuberlulofeblatt

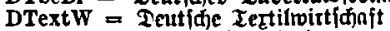

DTheol - Deutiffe Ibeologie

DTkZ = Deut f́fe Tonlüntllex-8cituun

DTLW = Deutide Terfinil in ber Laub wirtidjait

DTu = Deutífez Zurnen. Beitidrijt für rationaliozialiftilde sörper crzichung

DTW $=$ Deutidje Tednific 2uarte

$D t Z=$ Deutidje Beitidjrift

DUH - Deut/fingarifife scimlat. blätter

DuV = Diđtung unb Bortżtum

DUZ = Deutife uhrmađer-8eitun

DV = Deut ífes Boltätum

DVb $=$ Deut/dye Boltabilbung

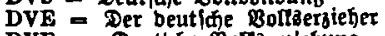

DVEg - Deut/de Boltaetztehung

DVerw = Deutífje Bertoaltuns

DVerwB $=$ Ter beut (id)e Berivaltungs beamte

DVerwBl $=$ Deutidje Betivaltunnos. blätter

DVerwK $=$ Deut\{aje Betwaltuitgä. Partei

DVj = Deut (đ)e Bterteljabrą)firift für Qiteraturivifieniajaft unb Beiftes.

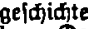

DVk $\Leftrightarrow$ Deutíge Boltâlunbe

DVN = Deutidie Bettehto-Nadicitert

DVoW = Die Deutíne Bogetrvelt

DVW $=$ Der Deutine Bollarotrt

DVw $=$ Deut (d)er Borlaroart

DVwsch 2 Dte beutidie Bollzinirtidjaft

DW $=$ Dermatologifije Modjen(firitit 
DWassW = Deutide Salfertoirtidiaft DWbl $=$ Deutífę æarmblut

DWehr - Deutifie $\mathfrak{B e h r}$

DWerb - Deutide Werbur

DWiss = Deutine whilīenidjaft. Olrbeit unb oufgabe

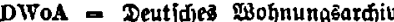

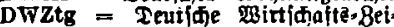
tung

DZ = Dematologif

DzaM o Deutíaje zahnärztliaje mlo natsijntift

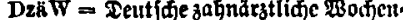
\{ditift

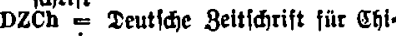
rurgie

DZggM = Deut jhe 8eitidtrift für bie aelamte getiditidie mebizin

DZH = Deutide 3eitifrift für \$om了. opathle

DZHk Deutíge 3abntieiltunbe

DZI = Die Deutífe Buderinbuftrie

DzKhk $\Rightarrow$ Deut /he Beitidrift für Steferbeillunbe

Dzak o Teutidie Babna, Munb. unb Fieferbeillunbe

DZNh - Deutidie Beitidirtit für Merventheillunbe

JZNeur $=$ Seutific Bett|cisift fü Neurologic

J)ZVStwkr = Deutfめe Beitiðrift fü Berbauungas unb EtoffroedifelPrariffelten

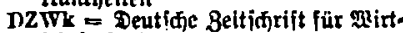
ifift tofumbe

DZWpf $\Rightarrow$ Deutidie Beitiđ)rilt lür Bofffaftszpflegc

$\mathbf{E}=$ Die Ciiel

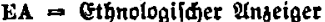

Fis $=$ Det Crbarzt, Reilage zunt Diut. fđien Arjteblatt

EtIm = Eliener 9lmanaf

FaPpA $=$ (5rgebrilfe ber allgemeinen Batfologie unb pathologifífer \&na* tomte

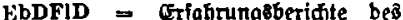
D. utfon Gluaretterbien it

EbHAW = Exfahrungaberidite bes Retdjamta fár $\mathbb{B}$ etterbienit

EC - Ftudes Carmélitalnes

EChO = Etgebniffe bet Eginurgic unb Drthopäble

EcHR E Economle History Review

Eck = Edart

Eckh = Edhart

EdImm $=$ Eraebnijie ber Jmmunitätă. forjकนurg

EeN = Ergebnifle ber eralten Ratur. ivifferífalt

EEnzF = CTgebnifie ber Enzbmfor. (f)ung

EFZo - Ergebnifie unb Fortifititte ber 8oologic
EGb $=$ Der Eriverbżgartenbau ver bunben mit Gartenbautolfien idiaft $\mathbf{E H Y}=$ (\$eidjarbts) Ergebniffe ber Sngiene, Balterlen. Imtmunitătz. forffiurs unb experimentelten Ihe ravte

ElMKh $=$ Etpebnifie ber inneren $\mathbb{R e}$ bizlit unb Rinberfiritumbe

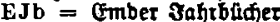

FEB = Eleltrifaje Siraltbetriebe uno Bafnet

Ekr (Endo) a Crtboltinologis

$\mathbf{E L}=\mathbf{E x}$ Llbrt

$\mathrm{ELBa}_{2}=$ Elettrifje Bafnen

EIBe $=$ Der eleltrifife Betriel

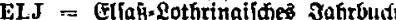

EINT (ENb) = GIeltri joje Madirifitell tedinit

Elsch (Esch) = Cfeftro[d]เveiß̧un!

ElW = Elettrizitätßżwirtiłafit

EMRVA = Entfdeibungent unb $\mathbb{R}_{i t}$,

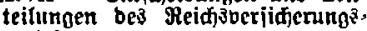
amteả

EmStr $\Rightarrow$ Cergebnifie ber mebitinifidien Strahlenforfdung

En = Gmergite

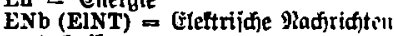
teginit

Endo (Ekr) $=$ Enbolrinologie

EphArch = Ephimeris Archailogik

EPhl = Ergebniffe ber :Bbiflologle

Ephlit $=$ Ephemerldes Ilturglcae

EPolAlm = Ethnopoliti(fier glimannd

ER = Guropäilíje Revue

Erk $=$ Crtenntnis

ErmlZ = Ermlänbiłळe Beitífrift

Ern $=$ Die Gmährung

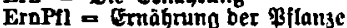

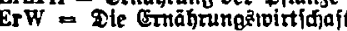

Erz $=$ Die Gratebung

ESA $=$ Enrasla Septentrionalls All. tiqus

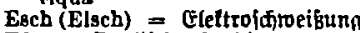

ESt = Crnplifóe Etubien

ET $=$ Giból unb Teet

Eth $=$ Ethos

ETheol - Evangelifife Ifeologie

EthoSt = Gthnologifífe Stubien

Etn a Etnolog (Ljubljana)

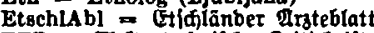

ETZ = Gleltrotedintíne Bettiकrift

EuFd = Guropähidjer ร̛̣mipredjbienit

Eultesp = Guropäił́de Geiprădie

Euli - Eleltrotednil uno graidiner, bau

Euph $=$ Guphorion

EuR = Curopäijo gevue

EvD = Evangeli\{hes Deutiđjlatb

EvErz = Dite Coangelifole Erziegung

EvKbls - Evangelifdes Sirdenblatt für Sditefien

EvMMI = Evangetij gaxim

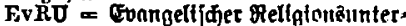
rifít 
FvS = Evartgeti (j)sozinl. Biertel.

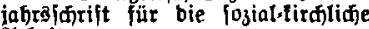
Qrtbeit

EvTheol $=$ Evangplijife Theologic

EWCM $=$ Gidier $\mathfrak{g n g}_{\text {u. Sie }}$ Rittei. lunger

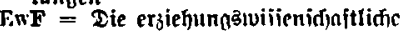
Froridung

$\mathbf{F A}=$ Foritardiv

$\mathrm{Fa}=$ Fauna arctica

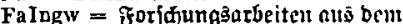
Gebiete bes Jngenieurivejen

FaOD = Forifiumgaratbeiten bes EGier batoriums Danzig

FArch $=$ Forftarfiv

FaSchwSchn = Forjđłungzarbciten für bas Eđ)weiben unb Edneiben

l'bGfb = Futterbau unb Gäriutter bereitung

FBot $=$ Fortlaritte ber Butarif

FBrPrG = Foridungen zur Branbelt. burailafelt uno Areupition (be= friidte

FchU $=$ Fettientijue Umidau

Fd = Foridungobienit

FDiözA = Freiburper Diözeian, Nrdjuiv

$\mathrm{FdR}=$ Fortlfiritte ber Möntgenologle

FEp = Fort (千) ritte ber Erbpatyologie

FermF = Fermentioriatuns

$\mathbf{F F}=$ Fajerfor $(\boldsymbol{d}) u n g$

$\mathbf{F g B l}=$ \&amiliengeiđjiđttliđje Slätter

FGH = Frantiurter (Ucographilid)

Jiriw $=$ Goridungen ans bem (b) bictc bca 3naenieuripelena

FGO = foridjungen sur Geifiditte ber Eptit

$\mathrm{HGP}=$ Fortidritte ber Geolonic unb galüontologie

Filst = Fortifiritte aul bem (Bebietc ber Böntgenftraflen

FGXst = fortiditite auf bem (seticte ber $x$. Etrahler

FH = Folla Haematologira

FI $=$ Forifjungsinintitute

FinA = Ftnanz-21rdjio

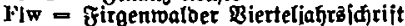
für Geologic unb Erbfunbe ber Eubetenlänber

FJud $=$ Forifumigen zur Jubenfranc

FL(FLw) $=$ Fortifiritte ber Eanbjuint (d) 0 it

Flkap $=$ Der Fruglapitäı

Flo = Flora obcr OIIgemeine botani idjc Beitung

FLw(FL) = Fortidjritte ber Lanbivirt: fitifit

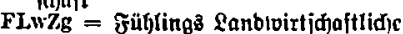
Beitung

FMed $=$ Frortifritte ber Mebiain

FMIn = Gortidntitte ber Mineralogie

FmPr = Eeinmedianil unb $\mathfrak{B r u ̈ z i f i o n ~}$

FN $a$ Fort/fritte ber Reurologie, Fipdiatrie unb threr Orrenzgebiete
FOd = Fortidgritte ber Srthobonti

Föt $=$ Fुbrbertedinit

$\mathrm{FoFo}=$ Forf(jungen unb fortid)ritto

Folbioth = Folla blotheoretica

Frad = Fundamenta Radiologica

PrHein = F̧ränlif(d)e Seimat

FrHj = Fräntilidie balbjałrşifirift

FriedGBI = ₹rtcbberper (Befairtits. b̆tätter

Frst $=$ Franjistanifकe Etubient

Frwd = Freipirtifinitliofez Yrojiu

FsTof $=$ Iremiefen unb Tonfilin

FT = Feuetungstechnit

FTh $=$ Gortidfitte ber Thernpic

FuA = Freube unb शrbeit

$\mathrm{FuB}=$ Film und Bilb

Fus = Fette unb Geifen. 9kuc folloc

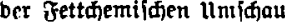

FVk = Forld)ungen zur Bortşfunto $F \mathbf{w}=$ Foritruitt

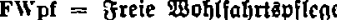

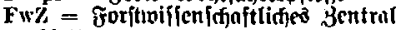
Glatt

FZool $=$ fortidrritte ber 3oolunic

FZP $=$ Franlfurter Beitfinrift fïr \$1 thologie

FRVR = Fiidiers Beitfdrift iiir Pler uvaltungostcdit

$\mathbf{a}=$ (Moetfic

FA = Geititige Rrueit

GAnn = Gcographildie Mntalcn

GAnz = Beographifder Orzeiger

G.10 = Û́. Ephthalmologie

Gart K = Bartentunit

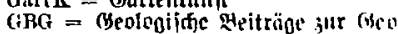
graphie

GBIXI = Gejujidfałblätter jïr Elabt unb Eanb Magbeburs

GbW = Ónttenbauvifienjđaft

Gbwirt = Ric (Hattenbaumintifiait

GdO = Geift bez Sitena

GdZ = Geift ber Beit

$\mathrm{Ge}=$ Genctiea

Geogr $J(b)=$ Geograpfi $\{$ des Jahrbud

GeogrW = Geographilde modjenidiriit

GeolAbh = Beolont idje Mbhanbluntect

Gcoll w = Geologie unb \$nuveicn

GcolR = Seologilaje Rurbldau

FcoMTh = Geograpfildje Mitteilultgcu

fïr Thüringen

GcotF = (iseoteltonlidje Foridunach

Gerlls = Merlands Beiträge jut Gco. עrytitil

Germ = (Berntania. Mnzeioer ber Rü.

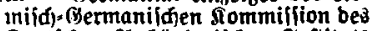

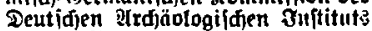

GermE $=$ Bermanenerbc

Germn $=$ Germanien

GerS = Der Geriđtșjaal

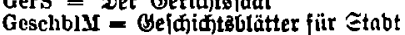
unb Lanb Nagbebur

GesD = Gejunbheitsbienjt 


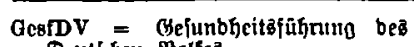
Diutiáen Bolfez

GesIng $=$ Belunbheit -Ingenicur (iesprW $=$ Dasె geforod)ene פ̧ort

GetrEck = Ter aetreue Gdart

(ietrT $=$ Getriebetednif

(iewRsch = Gewerblid)er oicd)tōj(juls unb urbeberredit

(iil = Bartenflora

Gg (Gt) = Der (Bemeinbetag

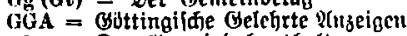

tha $=$ 2et (j)emeinoeljausfalt

GhFrh = Gebutşhilfe unb Fruten heilfunbe

Gless $=$ Dte Gieb́erei

(ilessBdPh = Giefencr Bcitrïge sur beutifien Qsidologic

GicssT $=$ (Sieberei-Ted)it

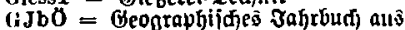
Diterreid)

(i) = Glotta

(ilaun = Ola fers̄ Plunalen iür Gelverbe uno Bautweien

GltB $=$ Mloštechifoge Berighte

(HtFB = Gleiztednif unb Faftbahubau

rilu = Ǵtüdaul. Serg* unb siitten. mätmifde Beit/dorifit

I.im = Die Gaz̄ntafe

(MNBE = Heologie ber 9)ecre uno bBinnengemuälfer

lin $=$ Einvuturt

VöN = GJttinger Radjridtent

(ioltdA (ASt R) $=$ Goltbammerz̉ Y(rđjio für Etrairedit uno Etrafprosel

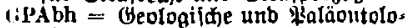
gifde ?tuhanblunget

(iPpsnF $=$ Gegenvartsurobtcute ber vigdintrifd)-reurologifipen For. (4)นกn

(ile = Ter (bolbente Keiter

GraphB $=$ Der Grawbiff)e Wetrieb

(iraphK (irK) = Tic (Brapfifd)en

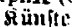

AiraphT $=$ (ifrublinide Ied)uif

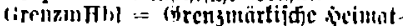
blätter

GrenzOL = Grenstanb Eberfaufits

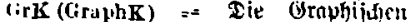
กünfte

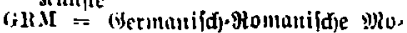
jlatsigitit

(inuch $=$ Grud)ot3 Bె Beitrüge jur $\mathrm{Er}$ lïuteruitg bes Deutiden Redits

LiJUR = Geduerblidier Reditsid)ui; unb Urfeberreft

(is) $=$ Germanoslavlei

(ist $=$ Tie Meitalt

lit (Gg) = Det Gemeinbetin]

liuA $=$ Glas unb rovatat

Guliz $=$ Gefurbfeit unb (Frziefun

GuL $=$ (oflasjidus und \&uftiduub

Liw $=$ Die Gartenjoclt

GWF = (jas. unb $\mathfrak{B a}$ alferiac

GW' = Beographifde $\mathfrak{k}$ odjeniffrift

Gymn $=$ Bsummotium
$\mathbf{G Z}=$ Geopraphifice Beitfigrift

GZZbl = Geologilides Bentralblatt

$\mathbf{H}=$ Helicon

HAb $=$ sollönbiidje Uffabentieberidjtc

$\mathrm{HAbh}=$ Qfbhanblungen ber Pffabemie ber $\mathfrak{x i f f e n i f j a j t e n , ~ S e i b e l b e r g ~}$

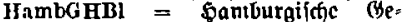

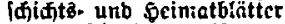

Harm = Die Sarmonila

$\mathrm{HBI}=$ \$iltorifð)e $\mathfrak{B}$ lätter

HBIVk = 5effifhe Blätter fiur 23olfs. luntbc

Hchl (Hochl) = \$od)[arto

HDIM = Mitteilumbet bes suupt vereins Deutidier Jngenteure Brün!

HeG $z=$ \$cfliifie Genteinbezcitu!n

HellL $=$ Dag \$eilige $\mathfrak{Z}$ anb

Helm = Die Seimat

Helml = Seimatreben

HeimSch = speintatidu\},

$\mathrm{Hel}(\mathrm{HL})=$ Selicnlanb

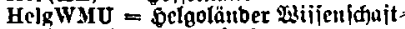

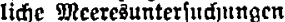

Herm $=$ 5ermez̉

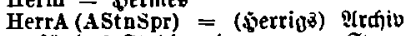
für baß̧ Єtubium ber thencren @pra. dien unb giteraturen

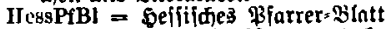

Hirte (ZHirT) $=$ Wodfircquensted)nit utio EIctiruafufti

$\mathrm{Hz}=$ seimatgaue

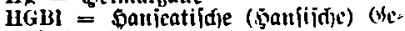
(if)id)tóblätter

HGPfl = Seil- unb Getuär3piflnujent

HII = Tie Sanbels̄ljodiffulc

HiL $=$ freintat int Bito, (bipiun

IIipp = sippotrates

HiW $=$ Die fimmelatwelt

IIJG = siftorifje 3abrlud sir Görrez-(Selellid)aft

HK = Ëine heilige Stirdje

$H_{L} L_{(\mathrm{Hel})}=$ Deiferlatib

HLE = 5ei zung unb Rüftutt!

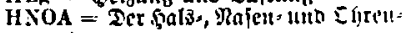
arat

Iloclil $($ Hchl) $=$ 5od)lanb

IIols = Sohenheimer giadizid)ten

Holz $=$ \$ol 3 , Zndfid)rift für sol serpurt unb gulghanbel

IIoSch =- Tie 5odidjulc

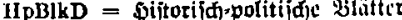
für bas latholifíge Deutldiland

IIPflub = Sinter Bilug unb Bitd

IIR = seimat unb oreid

IIRGZ = Janleatifide Bedits. UIID (S)erid)tz- Beit'́d)rift

HRW $=$ Dols als Moh, untb Qiserfitofi

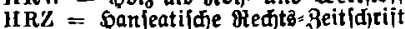

ISB = Eigurasberichte ber Ittabemic ber wiflen[d]aften, Seibelvers

USch $=$ Tie פofhere Sđjule

Hachm $=$ Der Suff(d)utieb

HSchS = Die höhtere Enfule für bett Frciitnat Endilen 
HechW (Hw) = \$odifjultollien, \$rag

HSp $=$ Der godffulfport

HStr a Die Sohe Gtrabe

H3Zphych (ZphlCh) = Soppe-Senter Beitiancilt fir phblologifie chemie

$\mathrm{HT}=$ Seetes-zedini

HoA = \$ofífute unb Qustanb

HuE = Sinmel unb Grbe

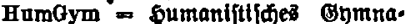
Thut

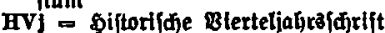

Hw (HschW) - Sodfifutritien

$\mathbf{H Z}=$ gijtorifaje Beit\{difit

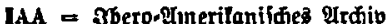

IAPhgr = Intemattonales 2 rafib für Bhotogrammetrie

$\mathrm{IBB}=$ Internationale Berowirtíaft unb Berateminil

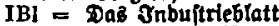

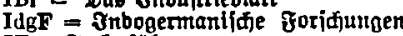

IF $=$ Imlerführer

IHgesch = Ingolftäbter Scimat geldidite

$1 \mathrm{ZZ}=$ Jnternationale sirdjliđie Bettiditift

InM - Imago Mund

IngA $=$ 3ngenteur.2urdit

InXiss $=$ Эntrere DRiffion

IPsT = 3nbuftrtelte $\mathfrak{B}$ (nd)oted)il

IR = Das Innere Peid

IRgHydr = Internationale Rebue ber gefamten subrobiologie unb shbro. grapbie

Isl = Islamlea

Islam $\Rightarrow$ Der 3 [lant

luSt - anbuftrie unb Èteuer

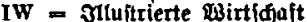

IZE - Ontemationale Beitifrift fiir Ergiebung

IZEw/ss $=$ Internationale Beit/drift für Cengtehungasmifienia)aft

IZ. = Internationales gentralblat futr Obrengeiflunbe unb ghino. Larungologie

$\mathbf{J A}_{\mathrm{A}}=$ Det Juttgargt

JbADR = Iahrbud ber פlabemie jür Deut/dies ORedit

JbAw = Jafreaberidjt fiut Urtertumo vilifenidialt

JbAWFDAF = צalirbud be Orbeit

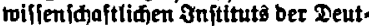
\{djen Arbeit3frout

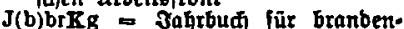
Jurgifde firdiengeifichte

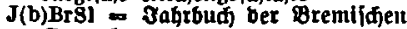
Eammlunger

J(b)DAI = Jaht6ud bes Deutíjen

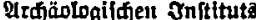

JbDDG $=$ उahrbud bet Deutidjent Dante-Befirildaft

$J(b) D Q=$ Xahrbud fâr Deutide Oe(ங)t市te
JbDLF = 3ahrou ber Deutionen \&uft. fahtfforfáung

J(b)diT = כahtoud ber braftlofet Eelegraphte

$J(b) E c h=$ Jafrobug ber Fteltrođiemic

JbElsLWG $=$ צaGrbud ber Elfaß:

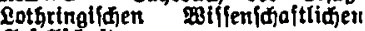
Beiellidaft

JbFDH $=$ Jahrbud bes Freten Deut. \{djen podiftifts (Eranlfurt/m.)

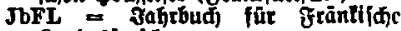
Lanbesforifiutr

JbGG = 3ahrbud ber (Joethe (Sejell fajait

JbGGH = 3ahrbud ber Geographi. fien Befiellialit sannover

JbGKSI (JOKGSl) - 3abrbilđjer für ble Gefitidite unb fultur ber Cla. went.

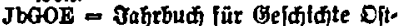
europas

JbHV - Yahrbud bes Dalleidelt Bet. banbes jur Erforfajung ber mittelbeutidien Bobenidiäse

$J(b) H W A=$ Jafrbuक ber 5amburger gBiffenidjaftidien Unitalten

$\mathrm{JbI}=$ Эahrbud 3mprtmatur

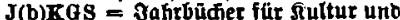
Geldidite ber Slaben

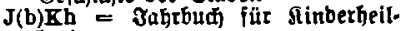
tunbe

$\mathbf{J}(b) k h S=$ aafrbud ber lunithiftori. ifjer Cammlungen in

JbKomw = Эahrbith ber fommunaltoilferifiaft

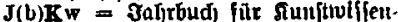
(d)aft

JblCh = Jah̆reşblätter fïr (Erirurgle

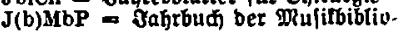
thet Beters

J(b)NadV = 3ahresberiat bes glieber. făfififí)en (seologilígen Bereins

J(b)NSt = 3ahrbuti fir Mationalóto. romle unb Etatifitil

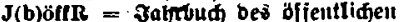
Meditz

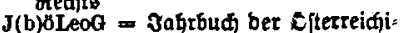

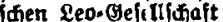

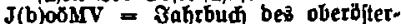
reidjifáen opulealvereins

JbPet $=$ उahrbud \$eter及

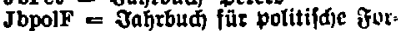
\{djung

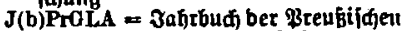
Beologilden Sanbezarifalt

J(b)prKa $=$ Jahtbud ber \$reufifajen surftammlungen

JbrKa $=$ Jahrbud für bratbenbur-

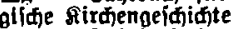

JBrSl = Jabrbud ber Breni(d)en Samminnen

JbSchbtechng (JbSchtG) $=$ Jahrbud ber Sdiflbautedint/djen Gelellídaft

$\mathrm{J}(\mathbf{b}) \mathbf{S K}=$ Jabrbud ber Sammiung fippenberg 
JbSoz = Jahrbud für Eoziologic

$\mathbf{J}$ (b)wBot $=$ Jaftbund)er für tolifen(quaftlidic Botanif

J(b)wpTZ = כahtbud ber solfienid)aft (idien unb praftifiden Tterzudit

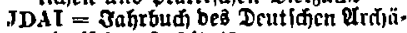
ologificien 3nitituts

JDG = כafrbud für Teutidie Ge.

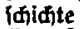

JdII $=$ उahrbud bar brahtlojen Tcle oraphie

JDMV \& Jahreaberidit ber Deutifien IRathematifer-Bereinigung

JEB $=$ Jahresberidit ber Erfurter 8iblinthelỏge fellifiaft

JEch = उahrbuh ber Eleltrodemte

JFDH = כafrbud bes Freien Deut.

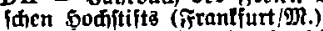

JFHE = Tahreşberidite ber Forftid)en \odjidiule Gbersitoalbe

JGenPs = Journal of Gencral Psycho$\log y$

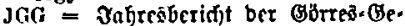
icllidjalt

JGGH $=$ Jahrbudi) ber (Seographi\{d\}en (blefellidjaft . batnober

JGIS = Journal Greater Indla Society

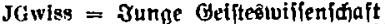

Jher $J(b)=$ Jhering 3 Эaf)rbüder für bie Dogntatif bes bütgetlidien Rerito

JhCAO VPrOI = Jahrcäfefte ber Go. iellid)aft für $\mathfrak{U}$ (nthropologie, urge: ifidite unb Bollatunbe ber Preus. Eberlaulib

JhHSA = כuhreşheite be focimat. bunbes Finevilts Endjen und Lanb ilnbalt

JhÖAI $=$ उalireshefte bes Ofterreini.

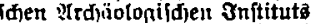

JIIWA $=$ Jahrbuळ ber Samburger Wifien ifinftlidien PInftalten

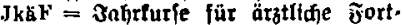
bilbung

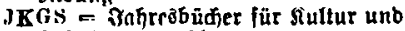
Beifidite ber Glaben

$\mathbf{J K h}=$ Jahrbü für sinberheillunbe

Jkh $S=$ Sohrbuf, ber lunitfiftoriffien Eamuthnnen it Micn

JK $\mathbf{w}=$ Jahrbud für sunitwilfenfd\}alt

$\mathbf{J L w}=$ 3oumal für Sanbiotrtidjaft

JMI $=$ Journal for Mathematlcs

JMbP = Jahrbudj ber Mufilbibliotfel Beter及

JMorheV = 3ahreabctiffte unb Mit. teifungen bes obertheinildjen neo lopiffien 23eteina

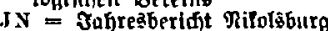

JNDW $=$ Tofreaberdate ber Rut. genteinfornit ber Deutidien $\mathfrak{B}$ iffen. fúft

JNGH - Zafreşberidt ber Maturfor. finenben Befellifiaft, Sarnober

JNeGV = Jalhreäberi(t)! bez Mieber-

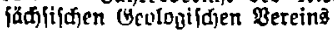

JNSt $=$ 3afrбu耳 für Mationalolonomie unb Etatlftil

JōffR = כahrbuต bes offentliaten Rechta

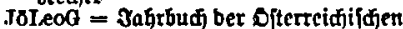
Leo-Brellifiaft

JoG = Journal of Ceology

JoIBS $=$ Journal of the Indian Botanlcal Soclety

Jom = Xoungెbura. Bgoltex unb Staaten in Diten unb Norben Curovas

Jobirv = Jahrbud bes oberofterreiकiificen Pulealveretrs

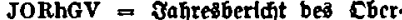
theintfonert Beologifíten Bereins

JOrn $\Rightarrow$ Fournal fir Emithologie

JpCh (JprCh) = Youmal für stalttidie chemte

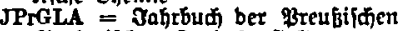
(Yeologifien Lanbezanttalt

Jprks = 3afrbuh ber Breubifinen funtifantmingen

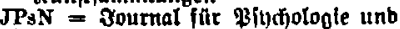
Sicurologie

JraM $=$ Joumal für reine untb ange, toanbte grathematit

JRAS - Journal of the Royal Aslatic: Soclety

JAAVMA $=$ Journal of the South Afrlcan Veterlnary Mfedical Aesoclation

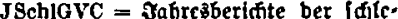

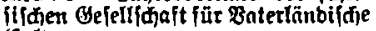
(sultur

J SchtG = 3ahrbud ber Sđiffbautedntifien Gejertifiaft

JSK $\approx$ Jahrbud ber Sammlung $\Re$ ip. penberg

JSW $=$ Jugenbifiniften-gaarte ber

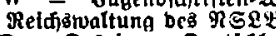

JuD = Das junge Deutífilanb

Jud $\boldsymbol{a}$ Judictun

Juew (Juwo) $=$ જtinertwofi

JuK1 = Tunge Iirdje. \$albinonats. fđirift für reformatorifijes chriften. tum

JurB1 = Surtittíge glättcr

JuRe $=$ Jupent unb Redit

JurR $=$ Juriftilifie Runbifina

JurW $=$ Juriftifle $\mathfrak{B o d i n f ( j r i f t}$

Just $\Rightarrow$ Die Tufti

Juwo (Jugw) = Zugenbrobht

JVm = જahteaberiđat füt Betcrinätmebifin

JVNkM $=$ Juhreŝberifite beş Beteills für Raturlunbe Manubeint

JVsthL $=$ Zahreşifirift fint ble Bor geiditdite ber fäd)\{ifd)-thüringl(d)en günber

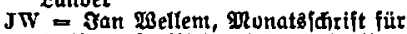
Dülfelborf, Rieberthein unb Berailías samb

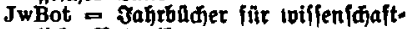
lidie Butanil 
JwDTZ = כafrbud ber wiffert(f)aft lidien unb praltílfen Tierzudit

JZNw = Jenalife Beitintijt für Mia turivif\{enfajaiten

$\mathbf{K}=$ Der Meil

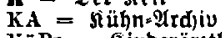

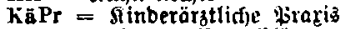

KatB] = Sinteffetifije biätter

KathFrb = Siatholisdje ซrrauelibilbutg in beutidien Bolfe

liathG = Der tatholijate Bebantic

KBI = stieler blätter

KchBh $=$ fiolloibdientilde Beifejtc

KdX $=$ gunft bet Mation

KDR = Sunft int Iritten (Icutid)en) शiei்)

$\mathbb{E} d V=$ Sunit ben $\mathfrak{B o l f}$

KEB = Sionititution unb ErGbiologie

KfF $=$ Sraftifahrioridum

KfMI = Solonialforitlide $\mathfrak{M l t t e i f u n g e n}$

$\mathbf{K}_{g}=$ sinbergarten

KGh = Famenzer Geidi(j) Bheitc

KiA = sirfie im Ongriff

KieHl $=$ গieler 8 lätter

Kiku = stirdienfunft

Kisozß3l $\Rightarrow$ Лirflidiojoziale slütter

KIV = תirdje im Boll

Klb] $=$ Sileruß̉blatt

KIF = Sileinafiatifice frorifungen

kIFb = salinifde Fortbiloung

KIMAh = Slinifdje gronatsblüter für Vlugentheilfumbe

KIMV WHL = Rieine Mitteilungen bes Bereins für \$alier, \$obelt. unb Iuftlytgicne

KITZ = Silepzios Textilycitidyit

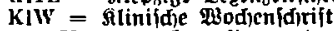

KM (Korr) $=$ Sitorrofiou und metall. (d)uts

KMF $=$ Micler @Reres̃[orid)unßcu

$\mathrm{KoBIGV}=$ sĩorefponbenjblatt Dis Gefamtvereing ber beutidien obs

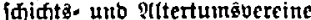

hoBlVndS = Siorreiponoensblatt bez Bereinz für nicberbentíde Evrad), foridjung

KolBIVsLk $=$ siorreiponocujblatt $b c$ ż Bercinş für fiebenbürgịj)e L'nnbes. lunbe

KollBh $=$ Solloib. Bcifeife

KollZ = solfoib- Beit(d)tift

KolR = Sioloniale Funbiafult

Jiorr $(\mathrm{KMI})=$ Sorrofiou unb gRctall iquis

KoSt $=$ Soloniule Etubieu

$\mathbf{K R}=$ Sartell-gunbidiau

KrA $=$ Die franlerhaugs? Yvothet:

KrBkL $=$ Sritildje Beridite jur tumit nelfjidtlidjen Qiteratur

$\mathrm{KrF}=$ Srantheitsfor [千]ung

Krim = Siriminaliftit

KritVj (VjGRw) $=$ Iritifije Biertel. jahtsàjifrift für Geletgelung unb Redtetzifieridant
KrMh = Ariminaliftilife Monatahefte

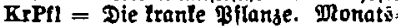

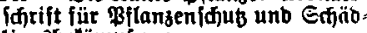
lintoủbelämpfung

KrSt = Ireio ber @täbte

KruppMh = S ruppidje פRonats̄feftc

KsozVj = Fölner \{bjtalpolitifíe $\mathfrak{B i c r}$. tetjafyrähelte

KSt $=$ Rantftubien

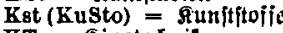

$\mathbf{K T}=$ Sinotedinil

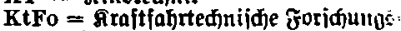
arbeiten

$K u=$ Tie Sunoc. Plitteilungäblätter ber Virbeitggemeinfdjaft für bie llr neidicinte Plorbiveftbeutiajlanbs

KuJu = Surit unb 3ugenb

$\mathbf{K u K 1}=$ Sunit unb Sirdje

Kult $=$ Die אiltur

KultT $=$ Der Fulturtef)nifer

KultV $=$ Die Sitturberjoaltıı!

Kunst $=$ Die Stunft

KuR = Siunft: Runbidau

KuSto (Kst) $=$ sunftítofie

Kuw $=$ Der Surfitwatberer

Kuwt $=$ Der Sunftwart

KVjhsoz $=$ Sïblner bierteljnhrabjejte für Eojiologie

KvSE = sali, vernumbte Gafje 1110 Grböl

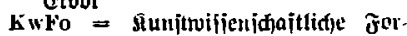
fdiungert

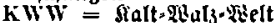

Ky $=$ sinrios

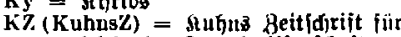
vergleidjenbe Evradmifienidjaft

$\mathbf{L}=$ Taß̉ giđt

J.A (Annch) $=$ (Liebigs) S(nnaleu ber Chemie

LaA (AkICh) $=$ (Yangenbed's) U(tr)iu für tlinildie chirurgie

I.aku = Tie Lanbestultur

LatIB1 = Qaucnburgildie Wlätter

I.blGRPh = Siteratutblatt fiit ger. nunifine und rumanifue Philologie

L.Ber $=$ Beridte ber Gädfifígen Vita. bemic ber 23 ilifenidiaften Leipzio

Lbls = Rel)terblatt Galjburs

LIBZ = Ecipsiger Hiencrafitult

J.ebo $=$ Der Yeberbuorm

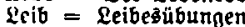

LeibkE (LükE) = Leibešübıแncn 1110 tisrpertique Erjieljung

Leop = Lcopoldina

LeopNa $($ NAL) $=$ Nova Acta Leupoldina

LErt = Lebentästiold

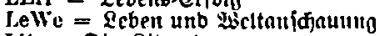

Lit $=$ Die Sitcratur

I.JBF = Lehrlinga $*$ Jugeub $=$ und $2 \mathrm{il}^{\prime}$, rufลี่uีrígrge

LKTVB $=$ Qeiphiner Jonjett $c$, Ifeater. uno Berfefré.:Blatt

$\mathbf{L L}=$ Qiturgifdjes̆ Leben 
LAI = Le Muséon

Lmasch $=$ Die Ranbmafofine

LMBI = 2üneburger SDufeumöblätter

Imod = Les Langues molleines

$\mathbf{L T}=$ giø)ttedinit

LThK = Qerilon für Theologic unb Sirdie

Lu = Xuthertum.

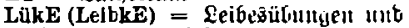
Iörperlidje Erziehung

LuF $=$ \&uitiabrtiforífun

LuJb = Quther.Jahtbu币

LuL = (id)t unb Lambe

LuMed = Euftiafrimebiziti

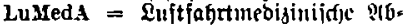
hanblungen

Iuth $=$ Quthertunt

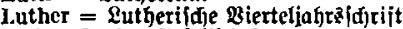
ber Luther-(Beirlifiaft

IuthJ(b) = 3ahrbug) ber Iutfer.(bc) lellidjaft

LuW $=$ Quftiafit unb Isifienlafait

Luw $=$ \&uftriffen

IVJSoEur $=$ Qeipjiger Bierteljubra. idrift für Eübofteutopa

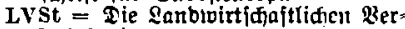
\{udfosftationeit

LwH = 2anbıintianafticie jodifiule

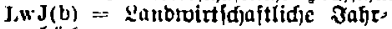
bïd)er

J.WPz Latbuirtidjnitlide Heljtier. alid)t

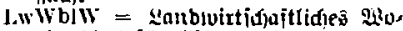
d)enblatt fïr Wö̈ttembcro

$\mathbf{L}=$ Shofrtos

Lzbl = Litcraritades Bentralblatt iür Teutidilutd

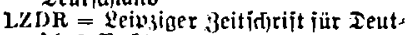
idies Vicdit

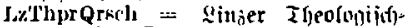
praltifide E.uartalitạrift

$M=$ Tic yulit

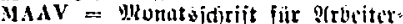
unb Prnaftelltelt.Beriogenung

Iacibl = Dlannfyeiner (Geidid)tôulät ter

SLAGW = 9)\}itteilunnen ber Antfro pologifien (Beicllidjaft gaten

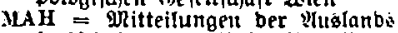
fodjifille an ber luiberfität $x_{c}$ criu

Malnz\% = Mainser Beitfdjrlft

MAIwVP = g)titteilungen ber 2(rbeites Intotvirtidjoftlider Berfud) ten Pran

Man = ykautus. Beitfffrijt jür 2orges (d)idyte

MandBI $=$ MPanrfyeinter (Sefdid)t Glätter

MLAnn = Mathentatifife OHnalen

MarbJ(b) = Darburger Jabrbud für funitroifientafaft

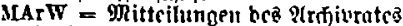
it Bien
Masb $=$ Mathematifoje Gemeitcr. berifite

MathB = Mathesls Brüssel

MaWe $=$ Martenloub unb mett: belverf

MBau = Traífinenbau

В[Ваu $/ \mathbf{B}=$ Mafáinenbau. Ier Betrić

IIBBG = פJttteilungen ber Bayerijaen Botantifien (Selellidialt

МBBI = Marburger Beiträge juแ Brinbenbilbungŝtucien

IBGGS = 9Ritteilungen ber Bulgari fajer Beographifacn Geiellfajaft Cofia

MBGL $=$ Ditteilungen ber \$abificu Geologi/fien Lanbezanitalt

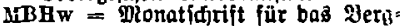
unb güttentwefen

MbleKch = Mitteilungø̋latt ber gaut: beşverbänbe evangelifaler firchell. hóre unb \$ofaunendjöre ber \$ro* vinz Gafien

MbleRU = Mtonatşblatt für ben Guan= aelifichen Reliaions

MblGPGA $=$ Monatß̧ lätter ber $(\mathrm{be}$. jelliciaft für \$ommeriळe Uejđidftc unb 2iltertumîtumbe

MbłHVH $=$ Mitteilungŝblätter bo Siltorifuen Bereilı für Defien

Mblstb $=$ Dronatżblätter für Єtrai fälfigenbetreuung unb CrmittlungsGilfe

MblvdSA = Dierlblütter für valfs.

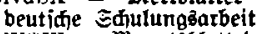

MblVGW = Monatablatt bes Jercius für Ö́fichiqte ber Gtabt $28 i e n$

$\operatorname{IBRA}=$ Mitteilungen ber $\mathfrak{B i o l o g i : ~}$ (d)en Meidjoanitalt

$\mathrm{MCh}=$ Monatobefte fiir Ghentic unb verwanbte Teilc anocter $\mathfrak{M i}$ iffetidinfteit

MchA = Mlikrochimica Acta

MLV $=$ Ditteifungen bes (Soppernitus̆ Bereinz für ßiffienja)uft unb sumit in Thorm

MDA $=$ Mitteifungcu ber Deut fajer शtrobentie

IDAIA (AthMitt) = Mitteilungen bia

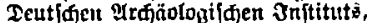
P(theni idje glbteilung

MDAIR (Kömsitt) = Nitteiluugat beg Deutiden 2rajöologliajen उit [titut $3, \mathbb{R}$ bmilidje gibteilung

MdBIVk $=$ Mittelbeutfdue Blütter fír Boliafuinbe

ILDDG = MRitteifunget ber Deutidien Dembrolonildien Gelellfaiaft

MDFI $=$ gitteilungen bes Deutidjen ชor(d) ${ }^{2}$ ng

MDGVR = Mitteilungen ber Deutjacll Beielliajaft für Bolferredit

MDGWK = Dritteilungen ber Deut, idjen Geleltiáaft ber $\mathfrak{W}$ ilfenjajaiten unb Sünite in ber tifficdiofloronlts ifien Metublit 
MDHb $=$ Mitteilungen ber Deutiden Бeereöbü円erei

M DHITS $=$ mittellungen bes Teutfifen Gauptberbanbes bcr 3nbuftrie Teplite @đönau

MDIAA = Mitteilungen bes Teutidicn Infitituts für ägทpttiðe elltertums. lunbe in Rairo

MLLA = Mitteilungen ber Deutidien Sanbrointidjaft - Befetlidiaft

MdMpA = פittetlungen ber Deutidjen inaterialorüfungäanftulten

MDOO $=$ Mitteilungest bet Deut\{djen Orient-Belellidiaft

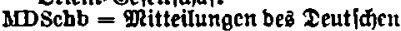
Efilllerbunbe?

MDStB $=$ Mitteilungen ber beutiden Etaats̄beainten mit 5ơ fifjulthilbung

MDStkW = Mtitteilungen ber Deut: finen Steinbrudftartei, grocinitclle Pien

SIdV $=$ Dittelbeutfaje Rolthelt

Me $=$ gledlenburg

MEGV = DRitteilungen bc Gifmader Seichidjt Buereins

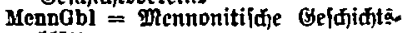
blätter

MErz = Der $\mathfrak{R u f i t e r s i e h e r ~}$

MetErz = Metall unb (Fr

MetZ = Mieteorologi \{áe Beitidrift

MFH - Mitteilutigen bes Fadjaus fđ̆ulfes für solbfragen

MTHG $=$ Dittellungen bet foreunbe beš humaniftlffien (S)m

MfleozAG $=$ פitteilungen ber floriftifiti.

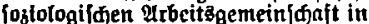
Rieberiagien

IlFww = פRitteilungen für Forftroirt. idaft unb For[troiffen/कiaft

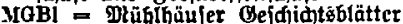

yGeolaW = Mitteilungent ber Esolo. gifinen Gefellfalit isien

MGeophWK $\mathrm{m}$ Mitteilungen ber Beo. bhnfitalifonen giarte siuniggber

MGFR a Mitteilungen ber (Bejellifiaft ber frreunbe Haabes

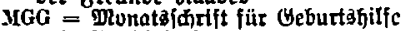
unb Sinnälotogie

MGOH = Mitteilungen ber Beograyhi. ficen Geleflifiaft samburg

MGGL = Mitteifungen ber Beographi. fíen Beiellfigat unb bes ratur

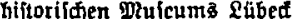

$\mathrm{NOGM}=$ Mitteilungen ber Geogra phifdien Befellidjaft in Miünden

MGgMCh (MGrenzg) = Mitteilungen nus ben Brenzoebicten ber Mebiain unb 何irurgie

MGGN $=$ Mitteilungen ber Geograuhiforen Befelifhajt Stüntbetg

MrGGTh = Mitteilungen ber Beogra. phifjen Belelt(d)aft filt Thüringen

MGQW $=$ Mitteilungen ber Beographi-

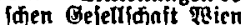

MGkK = Monatąifirift für Sottç bienft unb tirdilidje funft

MGKStg $=$ Mlitteifungen ber Beiell \{điaft für Gieler Gtabtgeidjidjte

MGMNw $=$ Mritteilungen zur Geífidite ber WReblzin unb Raturtviffenfifaften

MGrenzg (MrGiMCh) $=$ mitteilungen aus ben (Srenzgebteter ber Mebizin uno (Sfirurgie

MGTPB $=$ Mitteilungen ber Oelellidaft für Tierp\{ndologie

MGVk $=$ Mitteilungsblatt ber Gefelf. iđaft für Bỏlerfunbe

MGVsch = PRitteilumgen ber Befell.

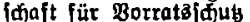

Yowi $=$ Magazin ber $\mathfrak{B i r t}_{\text {idja|t }}$

MH $=$ Mein Jeimatlanb

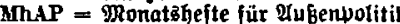

MhaPol $=$ Dlonat shcfte für auBื Politif

Mhch $=$ Monatshefte ber Chentic

MHDICSR $=$ QRitteilungen beg \$nupt. verbanbes Deut/fier 3ngenieure in ber Ccđjoltobalifuen $R$ Republit

WhfeV = MRittetfunacn ber helfilden famillengefídidtlidjen Bereiniguna IHI $=$ Qhitteilungen bes soerbiger. In 个titutz

MhßrPh $=$ Monatshefte für Muthematil unb Bhyfil

MhNSSP = Monatshefte für $\mathfrak{R}$, Gogialpolitil

MhnU = Dlonatefgeite für ben natur. tunblicien Unterridit

MhS $=$ Monatôfdrift für högere 巨̧̧ulen

MUStKrA = 9lkitteifungen aus bet Wamburgifđen Etaatsifranfcranitaltert

MHVFro $=$ Mitteilungen bes siftori ffielt Bereins Franlfurt/ Loer

MHWwA = Mitteilungen beş Santbur.

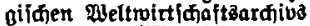

MigepalIR $=$ Mritteilungen aub bem Gcologíf(j-paläontologilfien $3 \pi$. ftitut ber Univerität Roftod

MlkrCh (ZMch) = $\mathfrak{M}$ ifrodjemie

MilchF $=$ Mildfitittdjaftlidje for ifiungen

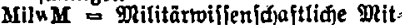
teifungen

MiMreGcLa $=$ Mitteilungen ats ber DReđlenburgi idjen Geologlídent Lan, besanitalt

$\operatorname{MinZ}=$ Minerva.Beitidrift

MIOG $=$ Ditteilungen bes Onitituts

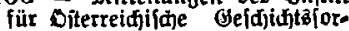
\{fung

MIsI = 9Mitteilungen ber 3alanbfreunbe

MIVSk = Mittelilungen ber Jnternationalen Bereinigung für Camen. tontrolle 
MVWSt $=$ Mitteilunger bes anter

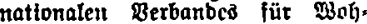
Iเungäweien unb Stäbtebau

Mnb $=$ Ditteilunger bez Jnitituta für $\mathfrak{W a}$ alferbau

JJIWR $=$ Mitteilungell brs כenapr

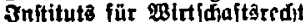

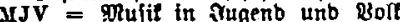

IKbG $=$ Pitteilurnen oce Friminnl biologilonen Beiellifiaft

Mkde $=$ Mrifeumblunbe

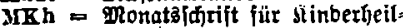
tunbe

MKhIF $=$ Mitteilungen bes̉ Sunit.

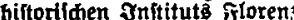

IIKI = Mebisinilate stinil

MKrb = Monntfकrift für Frebabe tänupiung

UKrbStr $=$ Monatō (đ) biologie unb Strafreditzreform

IIKrStr = Monatşđđrift für firiminal bindologie und Strafreditšreform

SLKWIEF $\Rightarrow$ MRitteilungen bes Saifer

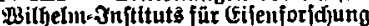

$\mathrm{ML}=$ Mitteifungen für bie sanbınint. idaft

MLN = Modern Language Notes

Mmech $=$ Die Mebizinmedianif

MMGSH = Qlitteifungen aus bem

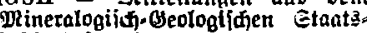
inititut Dambuts

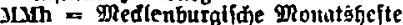

3nos = Tlundenter Mlofai?

XXPhMan $=$ פünthner Mufeum für Bbtlologie bes 9littelalters̄ unb bcr Renailfunce

MMschW = Mitteilungen aus ben Marlfófeibervejer

MMVL $=$ Mtitteilumgen bcs DiufeumBereinz it Raibam

MMW $=$ gRundiener Mlebiziniid;e Wodienifirift

SuatYNvpR $=$ Mitteilungen aus bem naturmilfenid)aftlidien Berein für Teuvorpon:mert uno Rügen

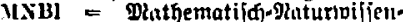

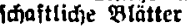

INNbVBW $=$ Mitteilumgen bes Morb bذhmlíncn Bereftiz für Seintat forid)ung unb 2 Banbervflege

INOBV = Pronatänađriđtent bca Ofterreiditifer getun.28creins

INVTr = Mitteilunnen bes glatur wilieniafaftliaen Bereins in Zrop pau

MOBstV = 9)itteilungent bes eîter reidjildien Berulaftenographenver banbes

MOIG = MRitteilungen bes citertelđi

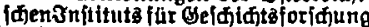

MOLFG = MUtteilunger ber Oiterretfilfoner Eanb- unb rostroitiof IIfien Ge\{ell[ð)aft
$\mathrm{MOh}=$ Monatöghrift für Ofrenfict. lunbe

MohaV = Mitteifungen bes Ever.

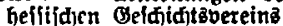

Molk7. = Mollesei-Beitung

IontR = Montaniftifice Otunbidjau

MorphJ(b) = Gegenbaurs Morpholo. gifunes 3alyrbud

Jotw $=$ Der Motorwagen

Mous $=$ Mouselon

IIPfI $=$ Die Thulitpflege

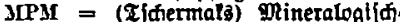
\$etrographiffe gritteilungen

MIPrAV = Mitteilungen ber BreuB̃iidien 2(rdiovervaltung

MPrKAWW = Mitteilungen ber Brä. hiftorlifien fommtli on ber ertabe. ntie ber giffe, idjaften wien

IMPS = Mittcilungen ber Mrovinj Endien

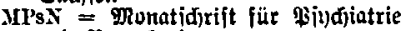
tinb Reurologie

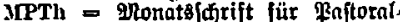
theologle

JQR = Mennonite Quarterly Revlew Mn $=$ Mitteilungen bes Rolanb, Dresben

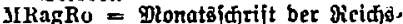

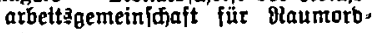
nung

MRAL = Mitteilungen bes Meidđânt für ganbesaufnahme

IIRB $=$ Mitteilunger ber Reiđsananitalt für Dobenforfduttg

YMDIV $=$ Mtitteilungen bes Meids verbanbea ber Teutiden פaffer. twirtifiaft

ING $=$ gittrifungen ber Naabe: (b) fellidjajt

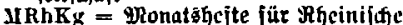
Sirúcengeifuidute

IRI $=$ Dtitteilungen ber Mofentfal Biolatoren o. nt. b. $\$$.

IIs = Monumenta Serics

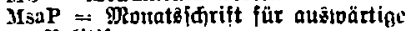
Bolitif

MSB $=$ Eikungâberiłte ber ganeri idjen ilfabemie ber wifienidjaiten. Dlündiell

IISchGPfl = Mitteilungōblatt be Ba Deut ifen Bereins füc Sfjulgefunbheitz. pflege

uschztg = Medlenburgifde sdulzeitung

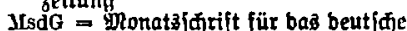
Beifteăleben

MSGV = Mitteilungen ber Sdjlejiid)elt Befellifiaft für Bolfalturbe

MSMIG = Mitteilungen ber Efinei rificet glufilforidienben Befelt. idjait

MSOS = Dtitteilungen bez Sentinata für Ertentalifoje Epradjet

Isp $=$ STutter\{prade 
MStGBl = Medlenburg.Streliber Be. (千) ‘)toblätter

MStwl $=$ mitteifungen ber Etern. twatte 8reslau

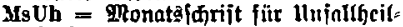
tunbe

$\mathrm{Mt}=$ Mreptednit

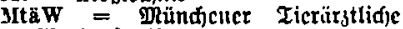

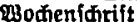

MTB $=$ MRelilanb Tertilberidite

MThBV = Dlitteilungen bes Thürinni. fđien Botarifífen Bereinz

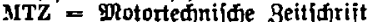

MUbG = Mitteilungett bes untortitiö, Bunbes (bobttingent

MCbAf (MUBM) $=$ Dritteilunacu bes Univer[ität3.:Bunbc⿱ 311 Mlarburg

MilJ (b) $=$ Mtündiner उabrbud . ber Gilbenben Sunft

MIuR $=$ gliffionarwif\{enifinait unb Reli gionồnilfentioft

MusGes = Muif unb Gelellid)ajt

MusH $=$ Ter Puítalientanoet

MusJV = Mufil in 3ugent uno 2bolf

Mus K $=$ gy unit unb stirche

Muswo $=$ Die PRufifwodie

MUV $=$ Mlonatşđfrift für llnjaltheil lunbe unb Berfiajerung s̄mebizin

$\mathrm{MuV}=\mathfrak{M k u}_{\text {it }}$ unb $\mathfrak{B o I t}$

MuW $=$ glarteniajus unb Wettbetucrb

IVASt $=$ Mitteifungett bed Bereins ber $A r s t e$ in Eteiermart

YVDH $=$ Dtitteilumnen bes Berbanbes Der Deutífen \$odidjulet

IIVGB $=$ Mitteilunnen beś Bercitto

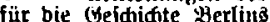

MVGDB = Mnitteilungen bes Bercinis für (Geirfid)te ber Ieutiden in Уölymen

SIVGE = PRittellunacn beß Bertins fï bie Beididite (Stfurto

MVGK $=\mathfrak{M}$ itteilungen ber Bercinigun! ber coroftefiflbefiber

MVGNm = Mitteilungent bej Bereinł für bie Geididite ber 'leumart

MrGoW $=$ g)kitteilungen bea Beteina

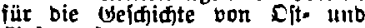
Beftureulient

MVGSTHHB = Mitteilument bes $2 \mathrm{ir}$ cins ber (Ueonraphie : Etubierenocn

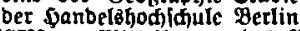

MVOUL $=$ 9:titteilungen beß Berein ber Beontaphen an ber Ilniverfitît Leipsio

MVGW $=9$ Mritteilungen beş Bbereinô für Gelaidte ber Etabt $\mathfrak{B}$ ien

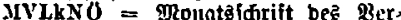
cins für sanbesturtoc voll glicber. diterreid

MVöZ1 $=$ Mritteilungen bes 23eteins Difterreidilidjer Beidientehrer

NW $\Rightarrow$ glebtrinishe welt

MWdark $=$ Mitteitungen ber $\mathfrak{W e j t}$ -

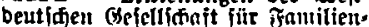
funbe
MWHK $\approx$ Mitteilungen ber $\mathfrak{B B i t t}$ teu bergijäen siftoriläen Sommiffiont

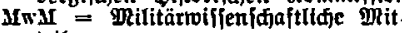
teilungett

MWo = Die Mebiziniide $\mathfrak{R o d j e}$

INR = MRilitärtwiffenidjaftlid)e Olunb idau

Mww = Metallwirtijjaft, MRetnlltviffen (djaft, Metalltedinil

MZ = Pratljentatifise 3eitidrit

MzoMB $=$ Mitteilungen bes Buolugi. \{dien Pufeums, Berliu

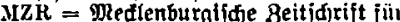

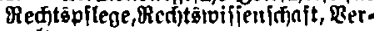
tvaltung

$N_{a}=$ Der Meroenar3t

NAädG = Vleues Ŷrdiv ber Geiell. (djaft für ältere beutjue Geidjiditos: lunbe

XAL (LeopNA) $=$ Sova Acta Leopol. dina

NAsäG $=$ शeucs श(rdfiv für jüdjiild) (Geldidite unb פIItertumbfunbe

NassAnn = Rafiauificte ?innalen

Nat $=$ Nature

Nats $=$ Naturiajub

NatSt $=$ Ration unb Etaat

NatW $=$ Die nationale Wuittidjaft

Nout $=$ Nauticus

$\mathbf{X B}=$ Reuez Sautentun

NBahnen (NBn) geue gahilu

$\mathrm{NBG}=$ Protik6latt bez sotnnifalen Gartenţ uID Muleums ju ĐerlinDahlem

NB!BhK = Sieujał)rớblätter bic sabi fajelt hipiturifden Siummilition

SuIDV $=$ Rad)ridjtenblatt für Toutjdi Borjeit

NolHGLA = Mutisblatt ber ociiiliden (Veologifofcu Lanbrizanitalt zu Ioru ftabt

NBu (NBahnen) = Picuc Hafunct

$\mathrm{NaJb}(\mathrm{JVndS}$ ) $=$ Jahrbud bez $\mathrm{B} \mathrm{er}$ eins für nicberbeutionc Eurat for [djung

NDK $=$ Meue Teutidie alinif

NaNsBBr $=$ glaturbentmalvilege แแn gaturfoub in Berlin unb 2 tranben Gurg

NDSch $=$ gieue beutidje $\Xi$ dutc

NdZV (NdZVk) $=$ Vieberbeutitic $3 \mathrm{rit}$ (firift für Boltôfultoc

$\mathrm{No}=$ Rorbelbingelt

NEEprT = Reuejte Eriinbungen vru tilfier Tednif

Neoph $=$ Neophilologus

NGQW = Mngirigten nout but Geiel iffnft ber æiffertdjaften ju (wit tinger

NGHG $=$ Radjridten ber viejiscule Sodjidulgeiellíifaft

NHbl = Qleue Geintatbtätter

NHJb = Reue acibelberger Jabrbünic

$\mathrm{xi}=$ Ntypen 
NiemZ (ZIntR) = Ylientet)er 3̧ Beit 佸)rift für intemationales Recht

$\mathrm{NJb}=$ (31berga) Reue Jaftbüffer jït bas flaififide QItertum, Geidjifite uliv. (Zortl.: Reue Bahtbüđ)er für Wilfenifaft unb Jugenbbilbung).

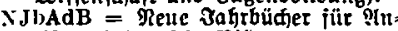
tife unb beutin)e gilbunt

YJbDW = Reuc ३ahrbücjer für Jcut

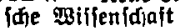

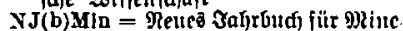
ralogie, Geologic unb भinläout ologii

$\mathrm{JbWJ}=\mathrm{T} \mathrm{Jb}$

NKZ = Meue Яirdjlide Beitidfrift

NL $=$ Die Relse \&iteratur

NLAL = Neues Saulitbiláes Mlunasin

NIM = Rieberlauliber Mitteilungen

NMbl $=$ Reues $\mathfrak{M u f}$ itblatt

Nitck $=$ Ter פeuc Merfur

NNsL' = Maquidjten alt Miebr: \{adjienz lltapejúidite

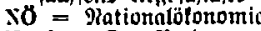

Nord $=$ Der Rorben

NordR = Mlorbildie Punbidina

NordV = Morbilific Melt

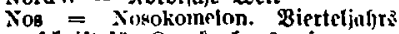

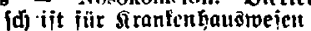

YPaid = Reur gäbngogifde Etubien

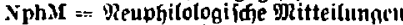

XphMs $=$ Peuphitologifac Monat idjritt

$\mathrm{Nu}=$ Tie Mille Minubidjau

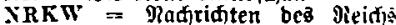

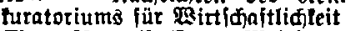

YRTh = Nouvelle Revue Thélogique

$\mathrm{NSa}=$ Mlieber\{adjien

TiäErz =- Plicberiänjiidirr Frsiejer

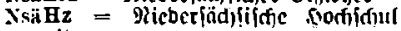
jeitura

$\mathrm{Ns} \|=02 \Xi=$ Hiricis

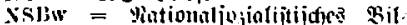
suntianucien

XSchR = Qleute Єduvcizer Runbitian

VSFrz $=$ Tor giationalipzinfiptictic (Etjleljer

NSrt $=$ Tie $n \approx$. Siemeinbe

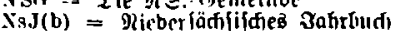

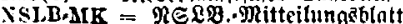

xsión - Mationalfojinlijiiffo Mounts. hefte

NSMGKS $=$ RS.'Mlitteilumnâblatt bra (raunmts fitr fommunalpolitit Sadicns

$\mathrm{NSpr}=$ Dic Theueren Epradicu

NSSP $=$ RS. Eoxialuolitit

NSt $=$ Rorbifde Etimment

SistgThK = Picue Etubielt zur (beiffidite ber Ifyelogie unb ber sitale

NTBIB = Nordisk Tldukrift for lbrk. ok Dibliotekswäser

$\mathbf{X T G}=$ Nederlandsch Tijdschrift voor Genceskunde

NThT $=$ Nifuw Theologisch Tljdschrift
NuA = Marfoje unb Onäjtgejie

NuG = Ratur unb Gelellifinit

NuM o Ratur unb Muleum

Num $=$ Muntiamati

Nus $=$ glatur unb Gqulc

ViIV $\Rightarrow$ Ratur unb Rotl

$\mathrm{NV}=$ Reuez Boll

$\mathrm{NW}=$ Rationale Birtidjajt

$N w=$ Die Raturvilienifiaitert

NWA = Reue $\mathfrak{R e g e ~ z u r ~ N i n t i f : ~}$

$\mathrm{NWe}=$ Teue $\mathfrak{W e g e}$

Nwith $=$ Maturtwifienjajajtlifjc glo matôncitc

NZArbR $=$ Reuc Beitjoriit ï̈r : $:$ beitärefit.

OasR = Eitafiatijaje Munbigan

OasZ = Ditaiiatildee Beitidrift

OChr $=$ Orlens Christianus

Od $=$ Ebal

OdMh = Ditbeutidje Mlonatibcitc

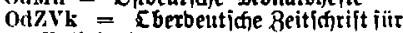
Roitakunbe

OE (Osteur) $=$ Sfteurova

OBauZ = Diterteiđifífe Bauzeitum

Obotz = Ofterreidifine sotanifale Beitifirit

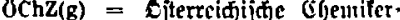
Beitung

ÖfGesD $=$ Difentlidjer Defuntbheit 3 . bienit

OhSch = Diterteidis hubhere Gdulc

OK $($ Ouk $)=$ Ol unb Rohle

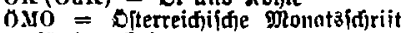
für ben srielt!

Oll $=$ Eiterreidifdje Runbitfau

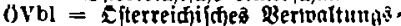
blatt

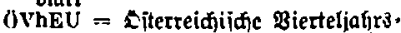
hefte für Grgiehung uno IInterridit

ovjFw $=$ Oiterreidifine Bierteliahr: idjrift für रुoritreient

OVw = Der Efterreidilid)e Boltabuirt

OZjurP = Diterreigilifies Bentralblatt fiir ble juriftiffie Brariz

oZöffR $=$ Opterreidiįdje Bettidrijt jür difentlidics Redit

OZVermW $(0 Z \mathrm{ZVm})=$ Oitertsidildic Bcitididift für Berne (jungžnelen

$62 \mathrm{Vm}$ (OZVermW) = Oiterreldiidie

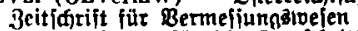

OFEW $=$ Srgant für ble fortlontittc boz Gijenbahniveienz

Offa (BMIvorgAK) = Effa. Berldite

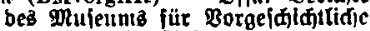
शiltertünice in Siel

$O G b=$ Det SGit- unb Gemületin

Oh $=$ Der Sitfeehanbel

OIB = Eberfaulitser $\mathfrak{B e i t r u ̄ o c}$

OlR = Nlympijue Runbjan

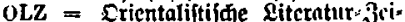
tung

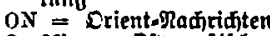

OprJth $=$ DiturentFif(d)c Moltatsfeite

Or $=$ Orlentalla 


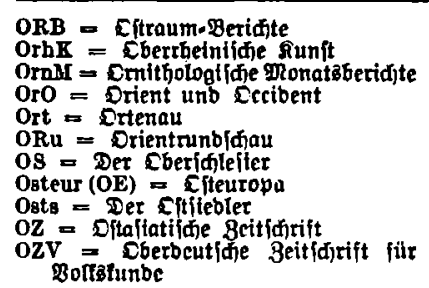

PadH = \$äbagogi|dje \$odjidjute

PädU = \$äbagogi(f)er ûmbrud

PadW $=$ Băbagogif́ne 2 arte

PadZbl = Bäbagogijăes Bentralblatt

Pajd $=$ Paldeums

Pal = Palaeontographica

PalA - Paläontologifoje QubGanblutben

Palae = Palaestra

PalJ(b) = \$aläftina-3ałrbud

PalZ = Maläontologi\{d\}e Beitid)rift

Panth = Pantheon

PapF $=$ Det Bablerfabrilant

Plphop = Bublifationen bes ajtro. phyitalifajen Eblernatoriums zu \$otabam

$\mathrm{Pb}=\mathbf{P a l \text { B̈oblologlcn }}$

PBB = Baul unb braunes geiträge zur (Geifidite ber bcutidien Eprafie und Literatur

Pbon = Pastor bonus

PetB = Fetermanns פitteitumgen aus

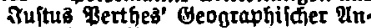
italt (jckt: Betermanns Beographi(d)e Ditteilungen)

Petr $=$ Betroleum

PtA = Bftügers Urđ̆iv für ole gefamte Bhntiologie

Pllar $=$ Die Bllanzenteale

PIIB (P11b) = 9flanzenbau

$\mathbf{P I M}=$ Pfälaifónes פRufeum

PIPrBl = Qjlälzijaes Pfatterblatt

$\mathrm{Ph}=$ Dle Bhrfil

Phanz $=$ philofophijajer Insetger

Phar = \$haru

PharmI $=$ Die Mfarmazentifoce $\mathfrak{I n}$ buftite

PharmZh (PhZh) a \$harmazeutifge Bentralgalle für Deut|hlanb

$\mathrm{PhB}=$ Bhylitalifo Bertate

PhCh - Bhnitil unb shemte

Phll $=$ Phllologas

Philu = Der philojophifłye untertajt

PhJ(b) = Bhilofophifíles Jahtbuक (ber Gortez-(Seleltidjaft)

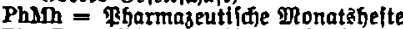

PhotFo = \$botograpfie unb Foridung

PhotInd = Bbotographilifie Inbultie

PhotRo = Bhotographlide sorreipon. bert,

Photogr $=$ Photogrammetrfa

PhR = Bhilologilone Runb/giau

PhBch = \$htlolophie unb Safule
PhWs $=$ gifilclogiicje Modienidjtift

Phypaz = Bhytopathologifice Beit (f) $x \mathrm{i}$ it

PhysR = Physlcal Review

PhZ $а$ Bgbiflalifdje 8eitidjrift

PhZg = Fhatmazeutifhe Beitung

PhZh (PharmZh) = Pharmazeutija Bentralhalie für Teutifilanb

PJZ = Brager Jurifti|d)e Bettidirift

Pl = Planta

PMA $=$ Proceedings of the Musical Association

PolBiol $=$ Politi|die Biologie

PolErz $=$ Folitifdie Erxiefung

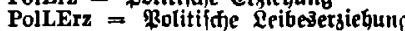

PomHpfl (PommH) $\Rightarrow$ Bommer|fic Seimatpflege

PomJb $=$ Bomtnter|dje Эabrbüळer

PommH (PomHpfl) $=$ Bommeridi Deimatpllege

Pr $=$ grotoplasina

PrBl = Broteftantenblatt

PrBw = Der pratti\{dje Betriebşinit

PrhZ = Frähilftoriidie Beitiditil

PrJb = Mreusijofe צafjoübier

PrKbl = Brattifíe farzinomblätter

$\mathrm{PrPs}=$ Irattifate $\mathfrak{B}$ indiologie

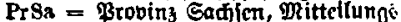
hlatt bes Sanbeşhauptmanna

PrSchPh = Praltlidie Edjul(ht)flt

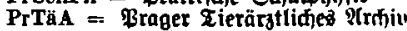

Prus = Prussla

PsArb $=$ Pindjologifaje Orbeiten

P\&Fo = Findologilde foríang

PsNW = $\$$ indiatrifi-meurologifac Bodieniditift

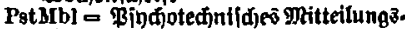
blatt

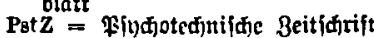

QDGB = Duellen unb Daritellunger zur Gefdidjte ber Burífienifiaft unb ber beut|afen Einheitßberoegung

QFIAB = Quellen unb forfoungen

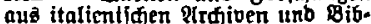
Itothelen

QSONwM = Duellen unb Stubten zur Beifidite bet Ratuttolifeni(f)aften unb ber Mebiain

Qub = Der Dutdborn

RA = Rour' Rrajio fár Entmidurang. medianit ber Srganismelt

RAB1 = Reigibarbettiblatt

Rad - Radiologica

RadR = Rabiologlidie Runbidiau

RABIH $=$ Beferatärateblatt für Seffent

RaI - Reale Academia d'Italla

RaMi = Maffe unb Mufit

Ras8 = Die Raffe

Rausk = Rechtoauatunft

RbSSchv $=$ Hedjenidjaftaberidit bes Samoäbtiaen Gatiterberetrs

RCP - Rasiegns del Comane dl Po. 
RDC = Rivista di diritto commercla] RdErz = Meidfazzitung beutifjer Er sietier

RDK $=$ Realletilon ber bcutidjen funtacidichte

RdT = Runbifiau Deutidier Tedinit

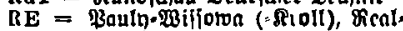
en3t)llopäbie

REI = Revre des Etudes Indocuro. péennes

REnt = Revista de Entomoloria

RepF $=$ Febbez Repertorium

RepKw $=$ Repertorium ber Siuritmifienifiaft

RegPr $=$ Bescarch and Propress

RF $=$ Momani|fje feridungen

Rfa $=$ Rumblunlardjiv.

RfRo = Raumforfdunt unb Raum orbnung

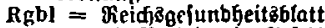

RGG = Tie Religion in B(ffild)te unb Begentoart

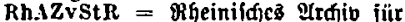
Bivil. unb Etraifreft

RHB $=$ Zet Riejer Seimatbote

RhBeob $==$ Pheinifjer Beobadter

RhBl = Rfjeini ide \&lätter

RhBINSKg $=$ Mheini间e slätter ber RS.: Rulturgemeitibe

RhH $=$ Mgeinilae weimatpflege

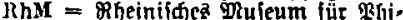
tologic

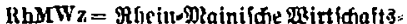
seitung

RhR $=$ Fficin unb Pufr

RhVBI = MY)einifúa Bierteliabrōblätter

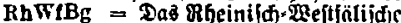
Sauncroerbe

RI $=$ Ier $\Re$ in

HIEBalk $=$ Revue Internationalc d'Etudes Balkanlques

RIEV = Rivista Internacionnl de los Estudios Vascos

RIOI $\cong R \mid$ Rista Indo-Graeco-Italica

RIISt $=$ Revue de l'Institut Intcrnational de Statistlque

Ik $=$ Die Neidjatirdje

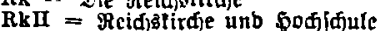

liKrf $=$ Das Redit bes Sifaftafyrers

RMal $=$ Rlvista dl Malarlologla

RMGIA = Revista Médica GermanoIbero-Americana

R MH = Revlsta Médlca de Hanburgo

RMI $=$ Revista Muslcale Italiana

RómMitt (IIDAIR) = פR itteilungenbe

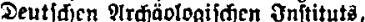
Ròntiłdje vibt.

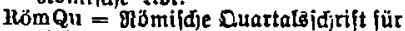
d)riftidide Qutertumahunbe

RPI $=$ reid aplanumg. Ergan ber Alabemie jür Lanbe日for|dung unb Feidjoblanung

RPr $=$ OABntoenuraria

RI' $=$ Revista de Pslhologie, Cluj
$\mathrm{RRn} \Rightarrow$ Redt bes Reidjżnäritanbes

RSchU = Riđutlinten für Sffroanger [djaft ţunterbred)ungett

nsnrv = Repertorium specierum novarum regol vegetablils. Bentral blatt zur Sammlung unb Beroffent. lidung von Belárelbungen neuer פîlanjen.

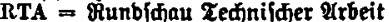

RTL $\Rightarrow$ Revio Technlque I.uxem. bourgeoise

RuH $=$ Ruler unb soter

RuhrA $=$ Der Ruhr-Prbetter

RuL $=$ Betá unb Länber

RuOm = Reld unb Eltmarl

Rvbl = greldobertoalturtobblat

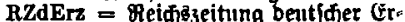
gle her

$\mathbf{R Z R W}=$ Miga[de Beitidjtift fïr Reditaroilien ifiaft

$\mathbf{S a}=$ Senckenbergiana

SaAn = Eadfien-Unthalt

SächsW = Die Gäđjitloje Qgittidjajt

$S a ̈ K B 1=$ Sählifífes fitcherblatt

SAGeschMed $\approx$ Subhoffa Ordjio fïr Beididite ber Mebizir

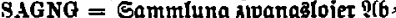
hamblungen aus bem Bebiet hor Rerven- unb Geilteslrantheiten

$\mathrm{SaJ}(\mathrm{b})$ = Saalburg-3abrbud

SavZ $=$ Beitifitift ber Eaptonn: tif . tung für Reditżaejuid,

SaVz = Eahiens Borzett

SBDmG = Sibungäberifite ber $\mathfrak{B} e r$ (ince Mtathemattifen Belellifialt

SbaNFB = Etburgaberidit ber Beiell:

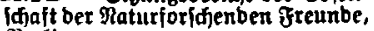
Berlin

SBphmSE (BPhmSE) $=$ Giłungâbe ridfte ber phoitaliid):mebizini(d)en Eocietät, Erlangen

SbVj $\Rightarrow$ Ë Ëebenbürgifłe Bierteljahr. iñrilt

$\mathrm{Sc}=$ Sclentla (Railanb)

SchA = Die Stjolle, P(nzbad)

SchAther = Gdjtoeizer Q1thiv für ๖e ralbit

SchAXb = Efulanzeiger fiur Mieberbannit

SchANPs - Eđjweiger Urđ̆iv für Res. rologie unb $B$ inditatrte

SchAT $\Rightarrow$ Sffrweizet Ulthiv für Tier, heillunbe

Schau = Śfauinalanb

SchBau $=$ Sđiffbau, Sdjiffaht uto Safenbau

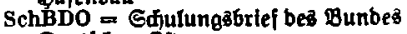
Deutidier Eiter

Schbf $=$ Gdjiffbau unb Gdiffahrt

SchBr $=$ Det Shulungobriei ber RSDथฌ.

SchBZ = Śftoeizetifije Bau-Beiturta

SchE = Equte unb coangelium

SchH $=$ Det Ediležnig.ફolfteinet 


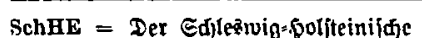
Erziebet

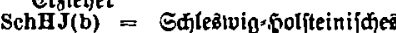
Эahrbu任

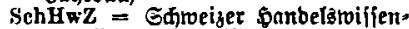

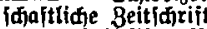

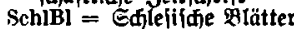

Schld $=$ Ghtooberlant, geitffrift bes Baucs Shruaben ber SDR i

SchlEI = Gdilngel unb Efijen

Schlern = Det Edlent

SchlOBl (Schlabl) $=$ Edilejiffie (Sc

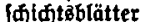

SchlH $=$ Edileiiīe weimrat

SchlMh = Stilefídje Mronat3̧rite

Schist $=$ Efflefithe Etimme

SchlStZg = Edjleíifje Eterographer jeitung

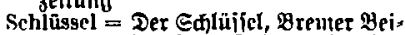
träne zur beutiden ภilutur unb झirt ifiaft

Schlupo = Shleif, untb Foliertednit

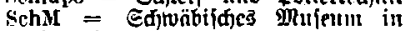

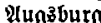

SchmiedA $=$ Edintiebebergs Profiv

schmollJ(b) = Edmollets Jahrbutd futr Gejebgebung, Berwaltung unt 23orfarvintifift im Deut ifien Reidje

SchMPM = Edtveizerifíe Mineralo. gifde unb Petrographific Mittei. lungen

SchMW $=$ Sdjueizer Mebijinilade wodjentafirift

SchMIZ $=$ Ediveizer Dlufit Beitung

SchNA = Eđmers, Rariofe, P̂rnäftheiic

schöH = Ediunere Eeimat

schPbl = Eđlefiidie Baítoralblätter

schraIK = 巨atrijten bes Beographi-

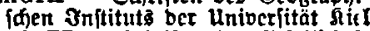

SchrGnFB = Edififten ber Geiellifiait naturforfकerber Freumbe zu gerlin

SchrHfLD $\Rightarrow$ Edfrifter ber podiffule jür Sebrerbilbung, Dortmunb

SchrHBK = Equiften ber STanbels * hodifdule fionigōberg $\mathfrak{P r}$.

Schrl = Tie Sd)rift

SchrKGG = Eđrtiten ber Rönigz: berger Gelefrten Geferifiofit

ShrNft+D = Edriften ber Maturfor. idenben Gelellifiaft Darzis

SchrPhOeGK = Edtiften ber $\mathfrak{B g}$ hit Palifif-olonomilaen Gejeltifialt sib. nigöberg

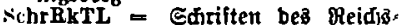
furatoriums für Teđinil in ber \&anb. virtidjaft

BchrVGB = Efiriften bes Bereinı für Geiditite bes gobenfeez

SchrVGL = SArriiten bes̉ Bercina jür bie Geiniate Seiphtos

SchrVNkU = Sđ̆riften bes Rereinş firr Raturlumbe luntertoeier

Schrvs - Ediriften bes Bereinß für Eptialbolitil

$\operatorname{SchSa}(\mathbf{S S A})=$ Das Ehüre Endien
SchSch = Efjouen unb Edjaffett

Schwa $=$ Effimaben

SchwBZ = Sdjweizerifice Bauzeiturn

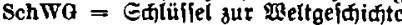

Schwld = Edimabentanb

SchwLwMh $\Rightarrow$ Effmeizer Lanbritt (d)aftliajc Monatohefte

SchwSchu = Gdineizer-Sdjule. .galb unonats̄idjrift für Grziehung uno Interrid)t

SchwWEW = Edjtoeizerijac Muajicr unb Energietvirt fojaft

SchwZVBSp = Sqmeizer Beitjoirit

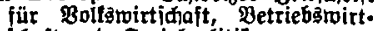
idjajt unb Eosialpolitil

SchZBwAE = Eqmeizer Beitiditiit fïr

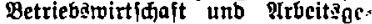
ftaltung

Sch7str $=$ Edjweizcrijde Beitidjrijt für Etrafredit

SchZuk = Edionere Bulunit

SchZVermW $\Rightarrow$ Ediveizet Beitjdirit für Bermefinngałvefent

ScPharm $=$ Scientia Pharmaceutlca

SD = Eubctenbeutifitum

SdAZ = Eüboeutique Qfpotffeterjcitul

SdMh = Eubbeutiojc Monatôneftc

SdUZ = Eubbeutidie Mhrntadicr $\cdot 3$ tn.

Seels = Der Seelporger

Seew $=$ Det @eetwart

SemBM $=$ Eemefterberidte RünIter

SemK = Seminarium Kondakovla. num ( Frag)

SFWo = Silva, Forfttidje 280 henjujrijt SGRNM = Gibungaberifte ber Defll: (d)aft zur Befóbrberung ber gelamters Maturwifienidafter, MRarburg

$\mathrm{Sgl}=$ Giegerlanb

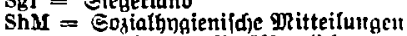
SienV = Giemenä-Beröffentlidungen nus bem Gebiete ber giadjridten. tedinil

$\sin =$ Sinicn

SIR = Elavifite Rumbifiau

SLwTz = Eubbeutids Sanbunittidjoit unb Tierkudt

So - Die Gonne

SocJus $=$ Social Justice

SodF = GüboftJeutidje Foridıunart

Bold $=$ eolbatentum

SozPr = Eosiale \$raris

SozZ $=$ Eobiale Bufurft

SpF = Epanifice fforiduuthell

SpfALV = Eaarblätjifie Mbjanto: lungen zur Lanbcs. unb Bolfafor. iquing.

Spl = Die Spiellđar

Spk = Dic Evatfafie

$8 p k F=$ Eparlafien-Fortbilbuugi: turiuza

SpKGE = Spreḑfaal für Serantil, अIas, Email

Sprk = Spradjlunbe

Sprkk = Spredjlunft unt Spredilunbe

sprS = Epredien unb Einget 
SpSchzg = Evebitions, unb S̈fiff, fahtag. -Beitung

$s p \%=$ Spebiteur = Beitifirit

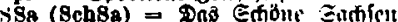

Siso $=$ Die Geelforac

$s t=G$ taub

StaB $=$ Dic Gtaatşbant

StadJJ(b) = Stäbel-a\}ahrbud\}

Stät $=$ Ter Gtäbtetan

StatR = Statiftifae Stunbiffau, Fray

StB $=$ Staflbau

stbeidN = ettubien beibrriets ber Rteberelbe

StBN = Studi Bizantini e Neoellenici StDHI = Studia et Documenta Hi. storlae et Iurls

StdZ = Etimmett ber Beit

StE a studi Etrusch

StEls - Etafi unb Elien

stEntZtg $=$ Gtettiner (Entomv(ugiid)c Beitung

StrkK $=$ Gtublen jur Beiđiøte unb Sultur bes nahen uno fermet Sftero

Sth = Gtrahlenthcrapic

St. Strb = Gtein-3nbuftrie unb Etein Gtrakenbau

St $I,=$ Stänbildies geben. Blätter fiir

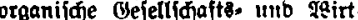
ifiaftalehre

Ntr $=$ Dle Straíc

itrB = Ter Etrab́enbau

StRein $\Rightarrow$ Die Etäbtereiniguu

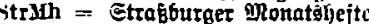

straV $=$ Etrafie unb Bertehr

strVk = Stubien zur religiofen Buffó. funbe

StSch (Stsch) $=$ Eteuctifjut:

StuK = Gtubien unb Fritifen

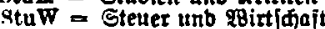

Stvgli $=$ Etublen sur verglciofenben Eiteraturge inifite

sud a Sudeta. Beitintift für gor unb grtibgeintontc

NudZVk $=$ Subetenbentidie 3ritiairift für Bottölunbe

SVaB $=$ Edirlften bes Bereins für Geifildte ber Baar in Donau ejujingen

SVaW = Squriften bes Bereins für Geiditute ber Stabt 28 ten

$\mathrm{SW}=$ Sleblums unb pirt hait

SWZ = Gaar-\$Bittidjaft

Synth = Snntbeie (Sollanb)

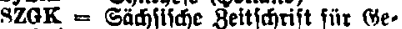
佸idjte unb hinit

$\mathbf{T}=$ Tie Tat

TA = Die tednilje âfijtentil

Tab $=$ Det Tabat

Tbc $=$ Dte Tubertulofe

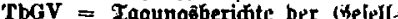
(d)aft iür Böllerturbe

TE $=$ Teçnijīe Erziefung

TeLn = Teđinil in ber Sanbluirtidjait

Truth $\approx$ Teuthonists

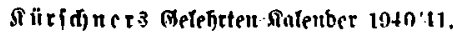

teannit

TGBl = Tefjnijde (Seneinbeblatt

Tha $=$ Thalassia

ThBl $=$ Iheotogiłđ)e Ylätter

Thd $=$ Therapie ber Gegentwart

'TheolR = Theologifice Ruttbidjall

ThF (ThorF) $=$ Daß Ihäringet fäbnleiı

ThFJ(b) = Tharnnoter Forfitiaties 3abrbud

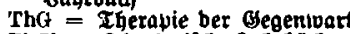

ThJb = Ifyeologifđe Jahrbüjer

ThLZ = Theologijufe \&iteratur- geitul 1

ThPA = Theologifíf-prattifife QRonnt fínift \$affau

ThpQu = Theologija:vraltijac Duar talfărift Qinz

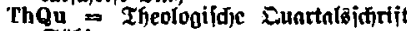
Iübingelt

ThR $=$ Theolugijde Revuc

ThSäZ (Th8Z(7) $=$ Thüringi id. Säd itidie Beitiơrift für (jelóftâte unb Sunit

ThistKr $\Rightarrow$ Zfeologitinc Etubien unt frritilen

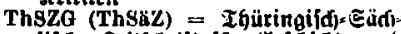
flide Beitifirilt fût Gejaidjte unb Sunit

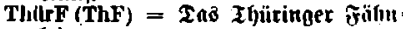
tein

ThuG $=$ Igeolugie unb Glaube

TlerI $=$ Die Iterertiährun

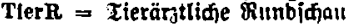

TirH $=$ Tirolet weimat

TirHein $=$ Etroler ఏeintatblätter

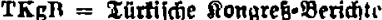

TMI = Tediniloge 9Ritteilungen

TMK(F) = Tedintidje Mittellunge1 Ftuivy, Foridumosberiकte

TMKT $=$ - Tediniłge Mittcilungen Sruvy, Tçintifie Seriaitc

TMIM $\Rightarrow$ Tedinifje gRitteifungen iiir QRalerei

TMTh $\Rightarrow$ Tedinific Medianit unk Thermobnnamil

Tolz (TIZg) = Toninbu|trie- Beitunn

TropPII $\Rightarrow$ Der Iroperupltanser

$\mathbf{T r Z}=$ Trierer gettífri it

TTE $=$ Teknlsk Tidskrift Ejektroteknik

TuBit = Teer unb $\mathfrak{B}$ itumen

TU $=$ Ter Tümet

TulBI $=$ Tabinger Blätter

TuM $=$ Túbinger Mitteilungtu

TuK $=$ Teđinit urb fultus

TuW $=$ Tedinil uno $\mathfrak{x i t t i \infty j a f t}$

Tvor $=$ Teatnil boran

ToRg $=$ Tijdschrift voor Rechts. geschledenis

$\mathrm{TW}=$ Die Tatwelt

TZpMI = Tcdini\{đjez Bentralblatt ïir prattifine Metallbearbeitunn

$\mathbf{U}=$ Tic ilmifau

UBIMN = unterriditablätter far Mlatljc matil unb Paturtwiffenidjaften 
UE $=$ unjer Figjājelo

UFo $=$ \&us untertidt unb æoridung

UH $=$ unlere £etmat

UM $=$ unjer $\mathfrak{D a}$ furenlanb

UPom $=$ unfer Bommerlano

$\mathrm{VW}=$ unjere welt

$T^{\prime} W_{0}=$ Die uhrmadjer-Wodic

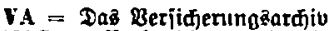

VAG $=$ Berkanblungen ber Plnatomiidjen Beiellifiałt

VARI = Beroffentlidungen bas offtro:

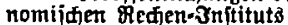

VBGK $=$ Berfiandlungen ber $\mathfrak{B a l t i}$ (j)en (beobätifín fommiffion

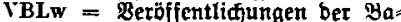
bijhen Lanbespterntuarte seibel berg. Sionigeftuht

VBVB = Berbanblungen bes Botani (đ)en Beteina ber Brobinz Bran. benburg

VBVR $(\mathrm{VbVr})=$ Rुöltcrbunb unb $23 \ddot{b l}$ terred)t

VI) $=$ Bolfatuın unb Tiftunt

VDGCh $=$ Berhanblungen ber Deut finen Belellliłaft für Chinurgie

V)GiM $=$ Berbanblungen ber Deuts

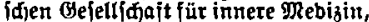
Bies̄baben

VDGKrF $=$ Berhanblungen ber Deut

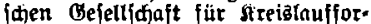
ifiuns

VDGR $=$ Berfandungen ber Deut fđien Gelellijaft für Maffentunbe

V DIK $=$ Berfanblungen bes Deutidient Znterniften-Gongrefies

vDKolUM $=\mathfrak{M}$ itteifungen bes Dcut (d)en folonial- und thber[ee 19 lu feums

v Dorth $=$ Betfanblungen ber Deut . fajen Orthopäbifigen (befellifiaft

VDPG $=$ Berganblungen ber Deut \{djer Bathologifdien (befellidjaft

VDPhG = Berhanblungen ber Dellt

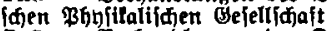

VDZoG = Berhanblungen ber Deut iquer "Boologífient Befellifjalt

VerkFo $=$ Bertehräwifien idaftlide Forifiungen

$\operatorname{VerkT}(\mathrm{VT})=$ Bertehrôted̆uif

VerkW $=$ Bertehrồwarte

Verp $=$ Berpadung

VerPr $=$ Die Berfituerungabraxtis

Verw $=$ Die Berwalturts

VerwA $=$ Bertwaltungäardjiv

Vest' $=$ Beftifie Beitínift

VevsozK $\triangleq$ Berkanblungen bes evart. geliffis fosialen gongrefíes

VFerd $=$ Betibfentlidiungent bez fer binanbeums

$V G=$ Bergangenteit uno Segentoart

VGdHNOA $=$ Berhanblungen ber Ge. fellf̧ajt beutifier salä. Rafen. unb Efren-Ftste
VGDNfA = Berbanblungen ber GH fellidjaft Deut|der Naturforidjer uno Arrate

VGeophIL $\Rightarrow$ Beriffentlifungen bee Geophylitalifíner 3nitituta Leipzis

VaGW $=$ Beröfientliqungen aus bem Bebiete bes Gefunbheitatwefers

VGKF = Berfanblungen ber Belelt

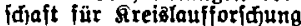

VGVStwKr = Berbanblungen ber (be:

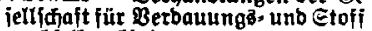
wehifeltrantheiten

VHfL $=$ Beröffentlidiungen ber fion ¡qujle für \&ehterbilbung

VhiGBk $=$ Berfanblutigen ber inter nationalen Gefellfijaft für Sobert lumbe

VhJb $=$ Beteritärffiftorifdes Jaf̧r buศ

VhMIH $=$ Beterinär-hiftorijąc Mittci lungen, \$annober

VHobG = Berbfientlidungen ber Sobbeas=(Belellifiaft

$\checkmark H S w=$ Berdffentliajungen aus bcII Seeres. Eanitätatuelen

VhYopf $=$ Berbanblungen bes hijto riffien Bereing für Eberpflals unb Megenţิburg

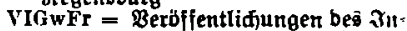
pitituts für Genolferiffaftsłoejen alt ber llniberfität f̧ franifurt $/ \mathfrak{M}$.

VIMk $=$ Beribffentlidiungen Deả $\mathbf{J}_{11}$. îtituts für Meeres̆tunbe

VIrchA $=$ Birdjowd Urdiv für patyo logildie Inatomie unb \$hnfiologie unb für lliniffe $\mathfrak{M}$ ebizin

VIVbBw $=$ Qeroffentlidurgen bex Internationalen Berbanbes für bas beruflide silbungatweien

VIVL $=$ Berhanblurgen ber Ynter tationalen Beteinigung für theore tifdje unb angetwanbte \&immologic

Viw $=$ Bolt im Werben

VjGR w (KritVj) = Aritifde Biertcl. jahrōid)rift für Befebgebung unb Oreditônillenifjaft

VjhKF $=$ Bierteljahrßheite sur Яิonjuntlutioridung

VjhstDR $=$ Bierteljabrăhefte zur Statif́til bes Deutínen Reidjes

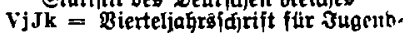
Punbe

VjLG $=$ Bierteljafrosłł frift ber_Luther (Gefellidjaft

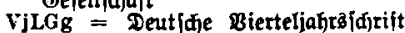
für giteraturtvifienidjaft unb Bci. iteogeifidite

VjPI $=$ Det 23ierjahreşplan

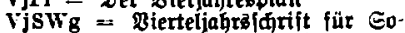

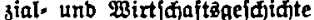

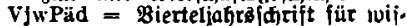
\{erijajaftlide ßäbagogit

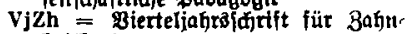
heillunbe

VkB $=$ Bolfătunblidje Bibtiographie 
VkwRW = Rerlehrimirtiđaftlidfc Ounb\{ajau, Wien

$v 1=$ Bogtlanto

ViwITHD = Beröfientlifumien aus bem Lanbrvirtidjaftliden Tnititut

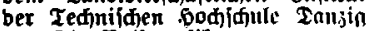
$V_{m}=$ Die Boltatuluit

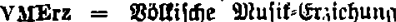

VMFI = Beröfientliofungett bes Duleum Ferbinanbeum in Эnnobntit YMIB $=$ Beroffenttidungen bez̉ glet: orologilgen Jnitituta, Berlin

YMLkL = Betobffentliduutaen bes $\mathfrak{M u}$ jeunz für \&änberlunbe, Leipzig

VTVB $=$ Berhatblunaen bes Natur hiftorifonen Bereins ber ßheinlande uno : Meftfalent, Boni

VYVBr = Berhanblungen bes yatur foridienben Bereing in Brïn

VöK $=$ Börtị̄e §ultur

vöKW $\Rightarrow$ Berobffentliajungen bes Diterreidjifífen fituratoriums iür EBirt' [haftlidjteit

FoKuR (VuKR) $=$ Boltstum tmb Sittur ber Bomanen

VorLeb (VpflLF) = Borratsyllege uito Lebenzimittel for \{dung

Yorp $=$ Der Borpolten

Vow ${ }_{4}=$ Bomt Maffer

VpfiLF (Vorleb) $=$ QBorratâpilege uno Sebensmitteliorfdunts

VPhYGW $=$ Berhandungert ber ybt

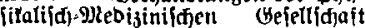
veürzbur:

VRa (VuRass) $=$ Bolf unb Rafí

VRAEF $=$ Beroffentltdumets Der Reidjzanjtalt für Grbbebenfor|d\}utg

Vsch $=$ Tic Zotssifule

VSchr :- ßolf unb Edrijt

Vuchw = Der Bottajautloart

Vsp $=$ Boltsipiegel

vStI = Beroffentliđungen ber Eternvarte $3 n n^{3}$ brud

VT (VerkT) $=$ Berlebratedinif

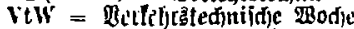

VuG = Bergangen heit unb Gegertwart

VuH - Bolt ult Scimat

VuKR (VoKuR) = Boffitum MIb Siultur ber Rontanen

VuR $=$ Bolt ttmb Reidi

Vultass (VRa) $=$ Bolt unt Haffic

VuSch $=$ Bolt umb Efiolic

VIStB $=-$ Bersfientlidjungen bor llniberfitüt = Etcrmoarte berlin $\mathrm{Ba}$. belaberg

VUStK $\Rightarrow$ Berijificntlid)utsen ber $u_{11}$

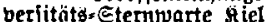

v väk $=$ Beröffentlidunngen ber Büter fullibe

VVSp $=$ Betganblungen bea Bereitt für Eozialpolitil

vVt $=$ Bolf uno Boltştum

vVVW $=$ Berdffentliáunger bes Deutidien Bereinz für Berlidie

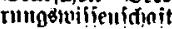

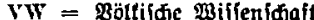

$\mathrm{Vz}=$ Der Bogetzug

VZBaln = Berofffentridungent ber Bentralftelle für salneologic

VZBGW = Berfanblungen ber 300s logila-Botanifinen Gefelliajalt, visien

VZG $=$ Berhanblunten ber Boolvgi: idjent Gefellifiaft

VzobotG = Berhanblumgen ber joolo:

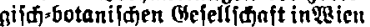

$W=\mathfrak{D a s}$ Wetter

$W_{i b}=$ Der פagent. Rübeder 3ahr\{ud)

WAbhDMPA $=$ Wifienidaftliđe wh: hanblungen ber Deutifien gate. rial- $\mathfrak{B r i f}$ ungazanitalten

WAbhNoK $=$ Wiffen\{fiaftlide $2 \mathfrak{b}$ : hanblungen ber Romtaleidunge. tommisfion Berlin

WAbhRAW $=$ wifienifiaftlide $\mathbf{9}(\mathrm{b}$. Ganblungett bes Reidsaintes für W3etterbienit

$\mathbf{W}_{\ddot{a}}=$ Tie $\mathfrak{m a ̈ r n t e}$

WA1M $=$ Wiener थrdjiv für innere Meoizin

WAL $=$ Wilfen[djaftlid,es êrdiv für Ianorotrtidaft

WAMA = $\mathfrak{B i}$ ifenfdjaftlide g(byanb. Iungen ber 刃aterialpriffungäämter

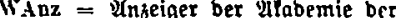
Wiilerifaften, æien

Was (WuSchu) $=$ weltarifiauun! unb Edule

Wassw $=$ Die Maliertintingit

WassWb $=$ Bzaffer: unb $\mathfrak{B}$ egebult3eitīitift

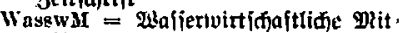
teilungen

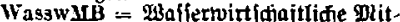
teilungen bes beutínen Micliorationsouerbatbes für 80 ótmen

WB $=$ Mertftatt unb Betricb

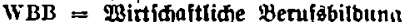

WHKKA $\Rightarrow$ Piener Peiträge zur Siunft unb Sulturgejothte Qlfiens

WbKw = Meinbou unb Melferwit, fifaft

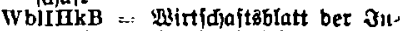
buftric- utib Sarbelolamiret 3॥ gerlin

TblLbHBr = भुonenblatt ber Lanbes.

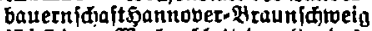

WblLbSA = \$odenblatt ber lanbe baurrtidiaft Gndilen-2(nhialt

WDGHl $=\mathfrak{W u ̈ z}$ burget Diöze\{all. gefdiditablatt

W Dst $=$ Mirt [đ]aft tōbien it

WdT $=$ Tie Belt ber Teditil

WdW $=$ Weftbeutídje Mirt\{đjafts. 3citung

$W_{e}=\mathfrak{D} \mathfrak{a}$ mett

Welbl $=$ Das̆ 2 einblatt

Weichs $=$ Beidflellanb

Wril = Dả Wieinlanb 
WestdBl = aBefibeutífe Blätter

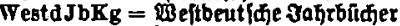
far Runitgefoitate

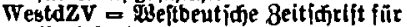
Bollohunbe

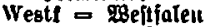

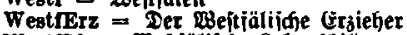

Westflb $=$ geitfälifíle Lebentabilber

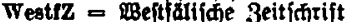

WestK $\Rightarrow$ Meftiote

Westm = Die Beftmiar

WoWs - Mefr unb Mafien

WF = Beftälifice Forfơnunget

WFS = Die खुebrttadt-Fad) (क)ule

$W_{g}=$ Die $\mathfrak{B}$ ertgemeinidjaft

WGesch $=$ Die Melt als Beifiditi

WI - Die 23 lt bes Xllant3

Wipru $=$ Der wittíajaltaprifer

WIR $=$ Det Mirtidjaft toring

$W_{1 W c}=$ millen unb gebr

WIWI = פgtffen unb gitrtent. gä̈ba. goglifice Beilage zu ben "mittet. Iunget aus bem höberen Gajul toefen".

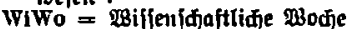

WJ(b)K $\mathrm{K} \Rightarrow$ Wtener उahrbud für Sunftgefinidite

WJbPhGW $=$ Whitienifjaftlinge Jahrez bertdite ber Bhtlofobhifiten Gejel! (d)ajt hit Noten

WK $=$ sas $\mathfrak{B e r l}$ bes fünitletş

WKbl c Wurttembergijies Surte. iporbenzblat

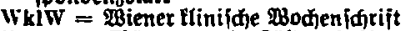

WKT $=$ mänue. unb \&älteted)nił

WkWw = Malfertraft unb walier. Ivirtidjaft

WLeb $=$ Weftiätijăe Rebenśbilber

$W I w Z_{\mathrm{B}}=$ Wiener Ianbwintidaltidit Beiturtg

$\mathrm{Vm} \rightarrow$ Die Rerfzeupmajdine

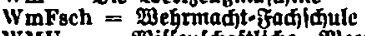

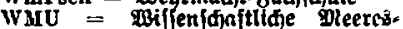
unterfutiungen

WmW $\Rightarrow$ Titence ntcbijiniffic IBodicn. iditit

WrapolG = 2uf bell अege zunt natio. natepolttifofen Ghinnafium

Wng (Wo) $=$ Die gofnnung

WNRR = MAtr(j)aftltaje Parjtidjten für guhr unb :

Wo (Wng) = Dte Mobrting

Wor = Der 9gormsgau

WoWl = Eotnung unb झirtidjait

$W p=$ Meltpoft

WPharmW = \$iener \$barutazeutilifie Bodjenfortift

WPZ $\Rightarrow$ gitenter Bräfiftorifđe ßeit. idjtift

WRH (Werft) = Werft, Heeberel, Safen

WRsgb $\Rightarrow$ Ier 9uanberer in Riefen= gebirge

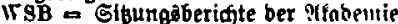
bet gisifenlafter, gien
Wrp = Mafferfport

WSt = פienet Stubien

Wateph $=$ Der 9 Seibenitephatte

WT = Mettitat tedint

WTaM = Mienter Zierärdtioge glunute fdifift

WTh (Wtrh) $=$ Der Wirtiafaftētrea bänber

WtMh a Wefitedintioge Monatşgeits

Wtrh (WTh) = Der פirtidjaftitrelt hänber

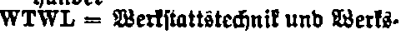
Ietter

WüBl $=$ ฌürttentergifaje Bunbeg blatter

WiDzGBI = marjburger Dibsejau.

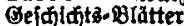

Württ = Măttemberg. Dlorats̄geiti

WüttSw = झüttembergijđie Sdjul marte

Wust $=$ Buirzburger Gtubieu

IVaStM $=\$ 9$ arjburger Etatiftiodi mitteilumgen

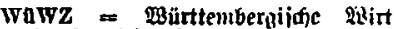
\{djajtz̧\}ett\{d\}rift

WuR = gaein unb Rebe

VuRV $=$ istrtidialt unb otedit oex Berfiquerung (Beiträge zur Beit. f(j)ift für Berjidierungstwe(en)

WuS = झborter unb cadien

Wusch = Menbe unb Ggau

WuSchu (WaS) = פBeltanffjauısı! unb Sdjule

WuT $=$ Bort unb Tat

WuWLt $\Rightarrow$ T3ährung unb osirtífujt

WVjhL $\Rightarrow$ wanttembergifale Biette!

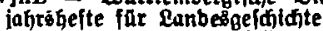

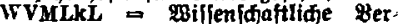
iffentldăungen bes $\mathbf{M n l e u m s}$ fïr In̈nberturtbe, Ielpgto

WVS (WVSW) $=$ \&BII enidjaftide BerDffentitifutgent bet Slementsmertc

Wvap $=$ Meltbertegripradjen

$W W=$ Bilfenfdjaft unb Weláneit

$W w=$ Meltrotrtiquaft

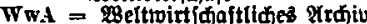

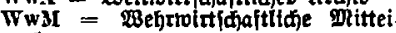
lungen

WwSpz $\Rightarrow$ Weatveiler für Spielzeng. fiorblvaren unb Sinbertvagen

WWZ = Mafier unb Megebau-geitfidrift

WrE $=$ Der $\mathfrak{W e g}$ zur Freihett

WZKM $=$ iBtenter Bettiditft fut bic Gumbe bes moraenlanbes

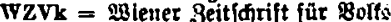
tunbe

YDS = Yearbook of verulatolos and Syphllology, Shicago

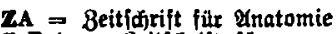

ZaBot $=$ Bettífitift fir angeluanoli: Potanif 
ZaCh $=$ Geitifint für angetwanbte Themie

ZAo $=$ Beitificift für Aithetit unb all: gemeine funitwilien iofaft

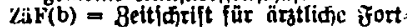
btlbung

LAEG $=$ Beitigrijt für Wnatomic unto

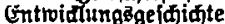

ZäM = Babratztlide Dritteilungen

ZaEnt $\Rightarrow$ geitfífrift für allgetvanbte Entomologie

ไïR = 3ałnärstlide Otunbidjau

ZAeSp $\rightarrow$ Beit\{quift für ägt)vtijac Spradje und Q̂ttertumblunbe

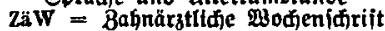

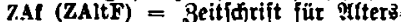
forífung

$\mathrm{ZaGph}=$ geitidjtift für angelvanbte Geovfingti

7.AGV = Beitifinift bes Marifiencr Ge (4)jidjtzoerein?

$\mathrm{ZAh}=$ Beitíhrift für 2ugentheiftunoc

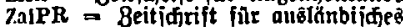
unb intemationales Bribatrectit

ZAkDR = Beitfintift ber Ntabentie für Deutiales Bredt

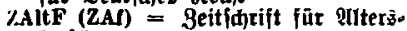
forforing

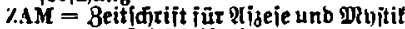

zaMet $\Rightarrow$ geitifirift für angemanote greteorologie

/aMIn = Beitidrift jüir angeluanbte Mineralogie

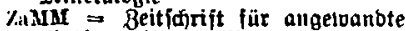
Drathematil unb gledianif

Zana]Ch $=$ 3eitidjrift für altalntifífo Stemie

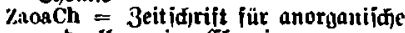
unb allgemeine Chemie

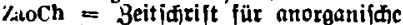
Ehemie

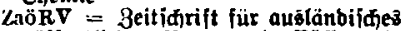
iffentlides Oledt unb golterrent

ZAPh $=8$ eitfidrift für R्ftroptinlit

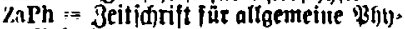
fiologie

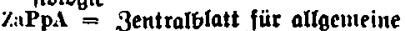
Patfologie unb patfologitige Tra. tomie

Zal's $\Rightarrow$ geit(a)rift für augetwanbte Bindotogie unb (charaltertunbe

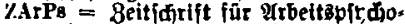
Iogie unb praftidie Plíndologie im allgemeinen

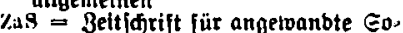
ziologie

ZAss $=$ gettjarift jür Mlifinriologic unb vertoanbte Gebiete

ZautMb $\Rightarrow$ Beitfđrift für nutogene $\mathfrak{M e}_{e}$, tallbearbeitung

ZAW = Beitiqurift für altteitamentlique Mifferifhaft

$/ R=$ Bentratblatt fur salteriologis, Baralitenlunbe unh rnipltionz tranthpiten
ZBaln $=$ Reitifuift für BaIneologic

ZBauw = Beit/anrift far Bautwelen

ZbasKG = Beitidirift für bayerifjic sirdjenge (d)tote

ZhayLG $=$ Beif(d)rift für Jayerījd) Lanbergef hidite

ZBDaR $=$ Beitíntift iür Beamten unb Behörbenangeitelltenred,

ZBFr $=$ 3eitidjift fur Büquerfreunic

ZBHS $=$ Beit farift für Berg. Sfitten unb Salinenweien im Deutifien greid

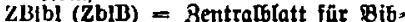
liothetsivelen

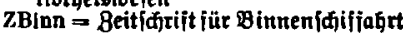

ZBiol = Beitfácift für Biologte

ZBK = Beitifintft fur gobestunbe unb FfTanzemernäbrung

ZbK $=$ Qeitidifift für bilbenbe Turit

$\mathrm{ZbLB}$ (ZBibl) $=$ Bentralblatt fat $\mathrm{bib}$ liothelawelen

ZblBv = Bentralblatt ber gauverival. turto

ZblG $=$ Bentralfitatt fî̉ Gsnnäfolonic

Zblah (ZblaHy) = Bentralblatt für (Getverbehggiene unb unjallberbü. tung

ZblGIIy $(\mathrm{ZblGh})=$ Bentralblatt jür Gerverbeghgiene unb unfalloerthi . tung

Zblgo = Bentralglatt für bie gejant: Dufthalmolonic uno ifre (Brent; aebiete

ZblHR = 3entralblatt für Đanbelşred)t

ZbliMI = 3entralblatt für innerc Debi: int

Zu]KJPK $=3$ entralblatt für M Meinticr lumbe unto Felztiertunbe

ZblPsth $=$ Bentralblatt für ił\{ydia. therapie

ZBot $=$ Beitiditift für Butanit

$\mathbf{2 B r}=$ Seit (firift für $\mathrm{Beraredt}$

ZBsch $\rightleftharpoons$ Beitififrift für Oinnenififf. fabrt

ZBStIA = 8eit|jitit bes \&ajeri\{ficu

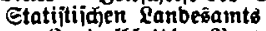

$Z B F==$ Bentralblatt ber Baubernaltitin $\mathrm{ZBW}=$ Beitiditift filt getriebsloirt. (d)ajt

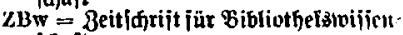
idjaft

$\mathrm{ZCCh}=$ Beitjogrift für Celfulofegentic

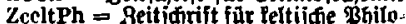
Iogie

ZChl $\triangle$ Bentralblatt für Chinurgie

ZChTh $=$ Bettiditit für chemothetapis

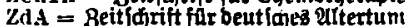
unb beutwe siteratur

$\mathrm{ZdB}=$ Beit|firift für Deutífje Bilbung

ZDesIab $=$ Bett jfitif ber Desinfeltoren unt Saborantert

$Z D A Q=$ Beitfinrift bex Deutifen Ben. logildien Geleflidiaft

7nGgesch = gettíntift iür bputífic

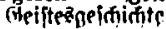


ZDGPh = Beitifirift ber Deut jøen Be. fell'́naft fir Bhotogrammetrie

ZDGwiss $\Rightarrow$ Beitfíntift für Deutføe Beiftestrilieninaft

ZDK $=$ Beitidrift für Teutidilunbe

ZdKPh = geitidirift für beutifie flut: turbhilopophie

ZDLO = Beitjđrift Deutider ¿lieb: Gaber, Drafefter

ZDMG = Bett|drift ber Deutiden MRor genlänbifden Befellidiaft

ZdöAV = Bettifitlit bes beutidicn unt

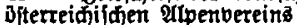

$\mathrm{ZdPh}=$ Beitidrift [ür beutine $\$$ Iogie

ZDPV $=$ Beitifirift bes Teutiden $\mathfrak{R} a$. Iättina

ZDVKW $=$ Betifinifit be Deutidien Bereing für ภunftrif\{en(क)ajt

$Z d Z P=$ Zeit狧rift für beutiden 3ivil= Droze $\mathrm{B}$

ZEch $=$ Beitidirift für (Elefitrodfentic unb angeroanbte phyfilatilde (She mie

ZeissN $=$ Beí-Nadfiditeıt

ZeitgSchr $=$ Beitgemäßc Ed)rit

Zeltw = Beitwenbe

ZEIV = Beitfítift bes cifelvereits

ZeII = Bett(d)rift für bie gefamte er perintentelte gheblzin

Len $=$ - 3ement

$\mathrm{ZePTh}=$ Beit(j)rift für eEverimentelle Bathologie unb Therapie

ZePhys = geit [́千juft für etberimentelle \$hy fit

ZErdk $=$ Beitiqurift für Grofunbe

ZErn : 3eitláfrift für (Ermäbrung

ZESpr = Beitidrift für Cingeboresten Epradien

ZEthn $=-3$ eitfđrilt für Githnolugic

ZevKM $=$ Beit\}átift für ebangeliface ริird)enmuli?

ZevRU $=$ Beit(j)ift für evangelilăen Religionzunterriot

$\mathrm{ZFb}=$ 3eitiđirift für Futterbau unb Gärfutterbereitung

ZFf Beitidrift für Fiermentforidung

ZFJw $=$ Beitifrift für Forit- unb 3agb welen

ZFMHy = Beitjørift für glei(d) unb mildibgiene

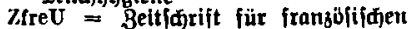
unb engliiøen unterriøt

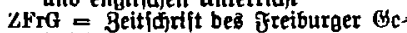

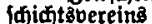

ZfrGuGW = Beitfđrift für bie freinvis lipe Geriđt verwaltung in $\mathfrak{B u ̈ r t t e m b e r g}$

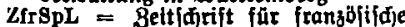
Sprafie unb Literatur

ZFTCh $=$ Beitfídrift far fatbent- unb Tettit-chemie

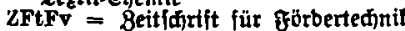
liub fraditoertebs
ZFTHB $=$ 3eitifirift ber Freunbe ber Tedinifien 5odifuule Berlin

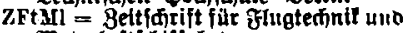
Motorluftidiffart

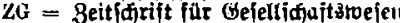

$\mathrm{ZgBrauw}=$ Beitjđịijt für bas gefautc Brautweien

ZGE $=$ Bcitid)nit ber Gejelljufaft fiur Crblunbe

ZGEB = Bettidjrift ber Griellifaft iür Erblunbe, Berlin

ZgeMI = Beitianrift für bie nejante cs perimcrtelle Mebizin

Zgenk $=$ Beitid)rift für gencinblid)e sulturvflege

Zgeogr (ZoGe) = Zoogeographica

ZGeogroW $=$ Beitjárift ber (Heograbhifdien Gejellodajt $\mathbb{B}$ ien

ZGeoMI $\Rightarrow$ geitfărift für (beomurbl)oro.

ZGeoP $=$ Beitífrifit jür Geopolitit

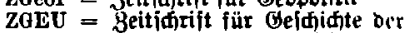
Grziefyung unb besె unterriđits

$\mathrm{ZgFw}=$ \{entralblatt für bag gefamte Foritweien

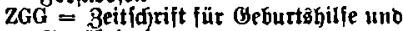
Gynuâtologie

$\mathrm{ZgGG}=$ geit/dgrift für bie pelamte Gunnälologie unb (Heburtôfillfe forvic Deren Orenzaebiete

ZgGisM $=$ 马eit任rift für bie gejante (Geridatliatie unb Eoziale Mebizin

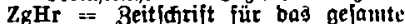
Sanbelöred)t

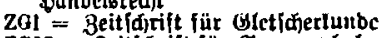

ZGM = Beit!市rift für Geomorphelogic

ZgNPs = Bentralblatt für bie gefantte Reurologie unb $\mathfrak{B}\{$ thdiatrie

ZGNsKg = Beitíatifit ber Beiellianait iiir gieberiädifíne Firdentaeidiaitc

$Z_{\mathrm{gNW}}=$ geit佸rift für bie gefamto Platurniliferidiaft

$\mathrm{zgO}=$ Zentralblatt für bie gejanttc Enhtbalmoloaie

ZGOrh = Beitífrift für bie Ocinidlc bes Ebertheins

ZGP = Beitlatift fït Beitüthıbe unt Bierbezudt

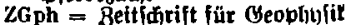

ZgphTh $=$ 8eitfigrift für bie gefautt: phylifalifíce Therapic

$\mathrm{ZgS}=$ Die Geitgemäre Gdirit

ZGshG = Beitidrift ber Gejellifjaft für

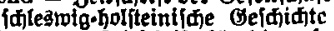

ZgStrW = Beit[hrift für bie gefa]tte Strafredjtstoifienidjaft

$\mathrm{Z}_{\mathrm{BStW}}=$ Geitidirift für bie gejautc Staatziviliferiataft

$Z_{\text {gTonI }}=$ Beitfđjrift für bie geiante Toninbuftrte

ZGTStH = geitiđjift für Gejunbheitô. teđinil umb Stäbtehygiene

$\mathrm{ZgTW}=$ Beitfajrift fiic bas gejamte Turbinenuefen 
ZQv = Beitifgrift für (Heiunbheit3̈ver * waltung

\%oVersW $=$ Beitidrift für bic gefamts Ber[idierungastotienidjaft

\%iyn = Bentralblatt fiit (G) nälologie

\%gZMK = Bentralblatt für bie gefamtc 3ahne, OMunb unb stieferheillunbe

ZHfrT (HfrtE) = 3eitlơrift für . .ood) frrguenztedinif unb Cfleftroafuftil

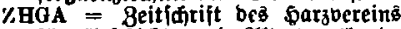
für (befigidite unb aitertumblumbe

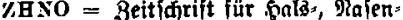
unb Chrenfeiltunbe

$\% \mathbf{H R}=$ Beitinifift für baz gefantic sanbles, unb Sonturziredit

ZHSchP = Bettiditift fitr sanbela idulpäbagogit

zHUk = Beitforift für seerco: unb uniformiturbe

ZHundF = Beitidrift für sunbcior

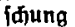

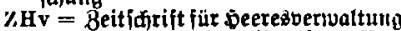

ZhVM = Beitififift bes hiftori ifen Ber eins Marbura (3ugollavien)

zhVSchw $=$ Beit\{jurift bes hiftorijđent Bereing fï̌ હfiraben unb Relt burn

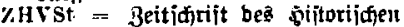
Rereinz für Steiennarl

Z.HW = Beitfifrift für baz seimat weim

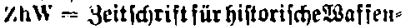
unb soitumlunbe

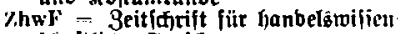
(diaitlid)e irorítiung

ZnuHp -.. Britígrift für Sanbels. wifienidjaft uno Sanbeläpracis

ZhygZSch = Reitlarift für bngieniidie Boolopie uno Gfidblingảbetämpfun

2Hylkr = 3eitid)rift für sngiene unb Inieltionalrantheiten

ZiAV] = Beit\{djrift für tubultibe $2 \mathfrak{d b}$ ftantınนกn

$\mathrm{ZJb}=$ Scitiqutiit für 3 nftrumentenbou

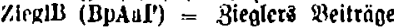
zur vathologitien Rnatontie unb 3112 allaemeirent Watholopic

ZII = 3citfd)rift für 3nbologie unb Traniftit

$z T k=$ Beitifirift fïr onjtnunenten tumbe

ZIIkr = Beitid)rijt für Jnfeltionäitrant Keiten, parajitäre Stanfigeiter unb Sogiene ber baustierc

ZiMI $=$ Bentralblatt für innere Mroiłin

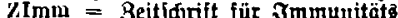
jurjdung unb erperintentelle Thera pic

ZIntR $($ Niem/ $)=$ (Niemeners) Beit

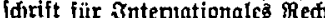

ZJJk $=8$ cit (d)rift jür Jugentolunbe

'jjurl' $=$ 3entralblatt fïr bie jurifitiote Firariz

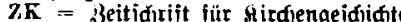

ZKf - Beitifirift ïir Sreh\&otidung
ZK fmV = Beitidrift für Ronjtitutionŝforidurg uno menlallife Bet: erbungstegre

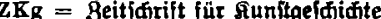

ZKh $=$ Beitforift für Sinberheillunbe

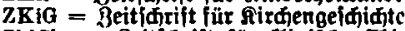

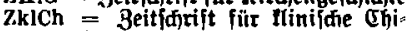
rurgie

ZklMI = 3eitiđjrijt für Zlini jđie MRebizin

ZKoL $=$ 及eiti币rift für Sonftitutions: Lebre

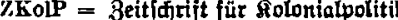

ZKrF = Beitifitift für §reislauffor. initing

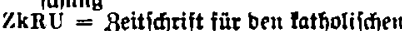
Melipionsunterridit

ZKrüF $=$ Qeiti币frift für grïbpelïür. jorbe

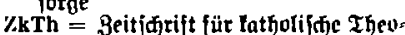
lonie

ZKultPs $=$ 3eitidgrifit iür Sulturyin. d)ologie

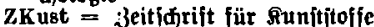

ZKuwiss $=$ Beitlifrift für Surort tvif\{enid\}aft

$\mathrm{ZL}=$ Beitingrift für Rarbngologic, Mhinologie unb ihre Grenzpebiete

ZLmasch = Beitictirift für Sanb. maiginen

ZlwkNs = Beitfifift ber Lanblvit ifiaftzlanmer für glieberlolefien

WMA = Beit|d\}rift für Morbhologie unb Arthropologie

ZmaF = Beit\{dytifit für utiltoflopiid\},

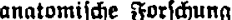

7.MB = Beitifirift für MRebizinalbeante ZMch (MIlkrCh) = Beitīrift für Mitro. diemie

\%Mlet $=$ Beitidirift iür Metalftunbe

ZMetw = Reitjdjrift für Metallivirtidjait

ZMin - Sentralblatt für Mineralogie

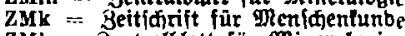

ZMI =- Bentralblatt für Pineralogic

ZnnU = Beitifirift für nathematifices uno naturtoifiemidaaitlidien linterridht aller Squulgattungen

ZMOe = Beit他ift für Mlorbhologir unb stologie ber Tiere

ZmpsF $=8$ eitjdrift für metapitudi The forfoung

ZMR $=$ Beitidurift für Mif́lionâtunbe unb Reltgionarvi\}fenichaft

ZMuaF (ZMundF) $=$ Bettifitit für Dunbartenfor\{fjung

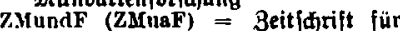
Mtunbartenforfdung

ZMus = Beitjóntit für glufi

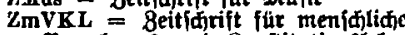
Bererbungä unb sonjtitutionalehr

ZMW = Beitfintift für gRiffionßwilien \{क人aft

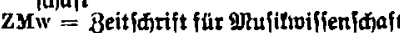

$\mathbf{Z N}=$ Beitidirift für Rationalotonomic

ZNeur = Beitíntift fâr bic gejamte Reurologie und pintiatrip 
ZNF $=$ Beltifirtif fur Mamensforfding ZNPs $=$ Bentralblatt für Neurologle unb rfodiatre

ZnsprU $=$ 8eitfartft fut ben uen: jorăblĭgen Untertiont

7NTKaK $\rightarrow$ Beltiditit fut Reues Teitament unb Sunft ber Olten Girdje

ZNW $=$ Beitidirit fur bie Reuteitas mentlidge פiffenfolt unb bie Rumbe ber älterm girdje

$\mathrm{ZNw}=8$ ettlíntft f. Slaturtoilienifiaft

$\angle O=$ Beitiatift für Otthopäbie

ZoA $=8$ oologiffier Inzeiger

ZoBeob a Boologifider Beobachter

YoBHV $=$ Beitifitift bes oberifjleitid̆en Berg- unb Suttenmäntifóden Berein’

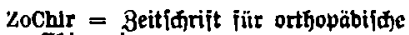
(EGinatgie

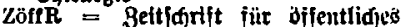
Redit

/offW $=$ Bettjartit für bijentlidge खittidat

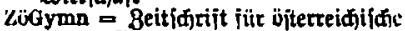
Sinmuafient

ZöLAV $=$ Bettīnrift bes öfterreinijigeut Treenimu - umb 9rofitetten-Bereina

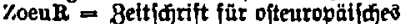
Pedt

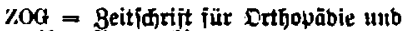
igre Erenzgebtete

$\mathrm{ZOO}=$ Der Boologifhe Garten

ZoGe (Zgeogr) $=$ Zoogeographica

$\mathbf{Z O h}=$ Beitfintft für Ehrenheiltunbc

ZoJ(b) = Boologifaje Jahrbatjer

ZONF $=$ Beitidifift füt Drtonamen. foridung

$\angle 00=$ Beitịd)rift für ophthalmologifd)c Dotil

Zool a Zoologica

ZOrg $\Rightarrow$ Beltifirift für Erganifation

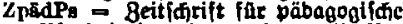

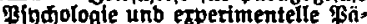
bagogit

zpddPJk (ZpPsJk) - Beitfarift far

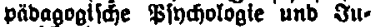
gemblunbe

ZPals = Beitidirift filr Paraftentunbe

ZPaPs = Beitiátift für ßarapipdjologle

ZPnb = Bettifititt füt Bflanzenbau

ZPHD $=$ Bettidrift für Bflangett. emäbrang, Dungung unb \$oben. tunbe

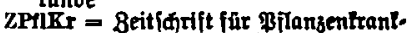
heiten unb isflanzenionut

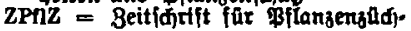
tung

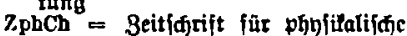
Shemie

ZphchU $=$ geitfartft für phbjilarifojen unb hemifinen unterrift

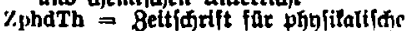
unb biätetiffie Theraple
ZphlCh (HBZphyCh) $=$ (Sopve- Seblet3) Beitidurift far phbliologifuje Ohemic

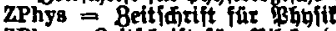
ZPK = Betifintift fuic Bilafunbe

ZPol = Bettichrift fir $\Re_{0}$ olit

ZpPsJk (ZpadPBJk) = Bett|firift für päbagogif包e Pindjologic unb כit: genolunbe

ZprG = Beitidjriit für praftifác (Sev. Iogie

ZpratL $=$ Beitidirift bes 93reuhi(d)cit Etatifitificen Qanbesamtes

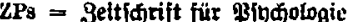

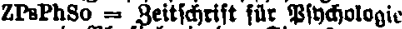

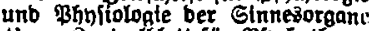

ZPsth = 3entralblatt fit Bindiothera. pie

$Z \mathbf{H}=$ Bafnärjtlldje \&unbifjau

ZRassK $=$ Beitidirift flr gafientunbc

ZRassph = Bett [dirtft fïr Raffer. phoftologic

$\mathrm{Zrb}=$ Buderribenbau

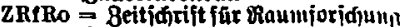
unb Maumorbmata

ZRG = Beitiditft far Reditŝgeididitc

ZRhF = Beitjđjrift für Rfjeunafor. idjung

ZRhVDH $=$ Beitidirift bes Rhetnijacı Bereinz fü Dentmatpflege unb Setmatidiub

ZRPh $=$ geitifitilt für Momantidje Bhilologie

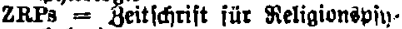
dotogie

ZRUF $\rightleftharpoons$ Bettiarift ber Feidjötelle fur ben Unterciatefilm

ZshugT $=$ Bettfordft für Gäugetier. tunbe

2Scha $=$ Beitifirit far Sojtuetgerifoje Beifidite

ZSchlHG = Bettidift frit Soflesroin. Dolfteinifide Geldjidte

ZSchm $=$ Beitiditti fir Sdulmuil

ZSchol = Beitiditit fir Gqulaitt

Zachst = Beltidirlft fis Gojpeifect;dic Statiftil und gollswotthidaft

zSchwz Bettiditft füt Gditoeine. sudit

ZSchz $=$ Beitionrift für Shafaudt

ZBexW = Betffictft fü Gezualtoiffen. idatt unb Sezualpolti

ZalPh $\Rightarrow$ Beitiontift für flatoifaje \$弓ji。 Iologie

ZSPhl $=$ geit(a)tift fur Stntteşphyfio. Iogie

ZSpIn $=8$ ettidjrift fũt GpirituBtrbuftrte

ZSRG(BavZ) - Beikifitift bet Savigrt). Stiftumg fut Rentzgeffichte

ZsTh $\Rightarrow$ Bettfirift far foftematific Theologie

Zstom = Beitíntift fũc Stomatologie, stert 
ZstP $(\mathrm{ZuP})=$ Bellitoii unb Bapier /sudG $=$ Beitifitit für jubetenbentine (Helditate

$\mathrm{ZsV}=$ Der Beitidriltenberleger

ZSW = geitfdgrift für Eozialmifienlidiait ZAVWL $=$ Beit|drift für GozinI: 1110 wirtidiaf tagefdidite

ZTbe $=$ 8ettiditift fur Tubertuloje

7TeFk = Beitidutft für Tiercmährun unb Futtermittellumbe

ztgW = Betturganoifien idait

ZThGA = Beltifintft bes 23ercins iiir

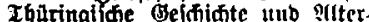
tumblunbe

\%ThK $=$ Beitfdift für Theologie unb Stirtic

/ThS = Beitidyrift für Ifperlogic unb Seeliorgc

zt:Ph $=$ 3eitifyrift iiir terfinituc Bhy it

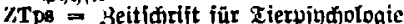

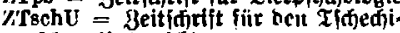
idien ulntertidit

ZTZa $=$ Beitfकrift für Xiersïntum unb Bühtungosbiologie

$\mathbf{z U}=$ Beitifindft für utrolonic

Zil = Det Budder

Zukk $=3$ Bưđutuntgồtunto

ZUL $=$ Betifingift fir lluteriunumg ber Lebensmittel

7uP (ZstP) = Beltitofi unb \&avicr

$\mathrm{ZuW}=$ Biel unb $\mathfrak{B e g}$

$\angle V=$ Beitung B B3erlag

$\%$ vBb $=$ Bahlungabertebr unb wane Setrieb

ZVBKI = Meitfdjrift bez 23ereinz wir liner saufleutc unb Tnbuitrieller

ZvdEV = Beitung bes Bereins bcut idicr Gijenbahnvertoaltungen

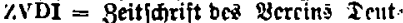
idier Ingentieure

ZVDZ = Bcitiđrift bes Bereins ber beutidies guderimbuitrie

$\%$ e $=$ Beitfáfift fiir Bolfzemüfrum

KverglPh = Zeitidrift fïr vergleiđenbe Whyliologie

TurglR = Beitidjift fite verglcidjento meditgonitienidiait

MorkW = Mcitifitift für Bctichra vilfertidja ît

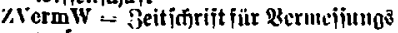
loeictt

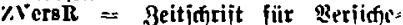

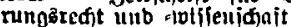

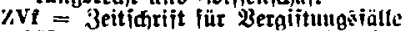

\%VaB $=$ Beitjurift bes Rercino für bie Geihidite Berlin

\%VGMSch $=-$ Beitfífiit bez Bercits iür bie Gcidichte smälyrens unt SAleltens

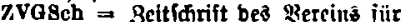
Geinidite Eqleitin's

ZVhG = Beitfírifit bes Pereins iiir hamburaijae Beificite

zVk = Beit/台rift für Bortäunbc
ZVKR = Meitidfrift für Boltätum unb Siultur ber Roinanen

ZVkw == Beit fí)rijt füt Berfełräwif\{en. idiaft

ZVKwlis = Beitfajriit beả Bereing fiir Sturitriilieniafaf

7VLAA = Beitídrift bca Rereinz fïr

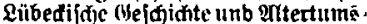
funbe

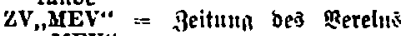
"MEV"

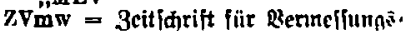
veien

ZVöR = Beit\}币itift für Balterredit

ZvPh $=$ 3eitfírift fiir vergleifient Bhtitiologic

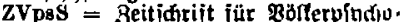
Iogie unb Eoziologic

ZVRbWVk = Zeit|fintift bes Berein

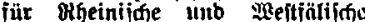
Bortstunbc

ZVSchHG $=3$ eitifitift bes Betein.

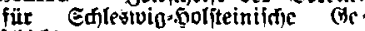
idjifite

\%vSpGISp $\Rightarrow$ Beitifitift iür verglei. genbe Eyradiforidung auf bell Gebiete ber inbonenumailiten Emradien

ZTthurG = Beitidiritt bez Serein:

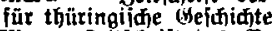

zVVk $=$ Beitfufrift hes Rerein fiir Borfstunbe

ZVW $=$ (Reuntantio) Beitifirift fiir Berifictunasiveien

$\mathrm{ZW}=$ Beitififift $\mathfrak{M}$ ürttember

$Z w d Y=-3 w i j$ jen ben Reiten

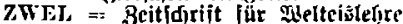

$\angle w F=$ Beitidfrift jiir tvirtidjaftlidse Firtinung

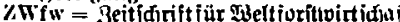

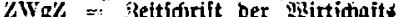
onuve 3uderinoujtric

ZWk (DZWk) - Beitfđqrift für wirt. idjaftätunoc

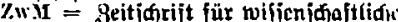
g)ifroffople utb mifroffoptínc Ted). nit

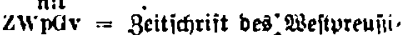

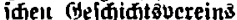

$\%$ Phot $\Rightarrow$ Beitidgritt für iviffenfduit. lidje \$hotographie

ZWIt = 3eitiafrift für odefrred)t

/ wiurttLG $=$ Beitiditift fât tointtem berpiinfe Ranbezzeidinte

$z W w=3$ citidrift fitr tveien

$\mathrm{ZwZ}=$ Beitidirift iïr wifienifiaftlidic 3oologic

$\mathbf{Z Z f}=$ Beit [đifift für Bellioriøung

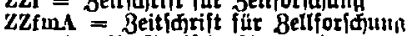
unb mitroftopifhe Unatomie

ZZIVP = Beitldirift fïr Bivilproseis

$Z \% \ddot{o}=$ Beitidurift fïr Bojle uno RPT. braữ̛̛̊ิfteuem

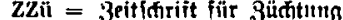


